\title{
Employment-adjusted Human Development Index
}

Hakan Mihci, Mehmet Tolga Taner, Bulent Sezen *

Abstract:

Purpose - The current HDI has a limited capacity to reflect the human condition and country rankings in an accurate way. In addition, the main critiques on the HDI suggest that it uses very few or perhaps the wrong indicators in measuring human development levels in countries. This paper aims to investigate whether the inclusion of employment as a criterion in the HDI would yield a different ranking of nations.

Design/methodology/approach - In this study, estimates of the proposed Employment-adjusted Human Development Index (E-HDI) are provided for seventy-seven countries for the 2000-2007 period, and comparisons are made both for changes in the human development index (HDI) and rankings over time in each country and for differences between the E-HDI and the UNDP's HDI across countries.

Findings - The experience of a relatively large number of 77 sample countries has offered promising results for the path to improve the current status of the HDI, and hence, to overcome its weakness in terms of ranking of countries. The additional indicator, i.e. employment, improves the explanatory power of the HDI and makes significant contributions to its reliability.

Research limitations/implications - The major limitation of the present study has been the lack of data for a number of the human development indicators for the rest of the countries in the world.

Practical implications - The employment-adjusted index has great potential to make the HDI more operational.

Social implications - By means of E-HDI, the human development performance of the countries can be better evaluated and compared with other countries by using additional information obtained from the employment position of their citizens.

Originality/value - This will be the first paper in the literature that incorporates employment into the HDI..

Keywords: Human Development Index, Employment, Development, Basic Needs, Human Security

JEL: O15, O57

DOI: $10.2478 / \mathrm{v} 10033-012-0020-8$

\section{Introduction}

The Basic Needs approach was a predictable consequence of studies on earlier views on development. The attainment of development had previously been evaluated in terms of growth maximization and industrialization in the early 1950's. Emphasis on employment as a new primary development objective evolved with time (Stewart, 1985).
* Hakan Mihci, Hacettepe University

E-mail: hakan@hacettepe.edu.tr

Mehmet Tolga Taner, Uskudar University E-mail: mehmettolga.taner@uskudar.edu.tr

Bulent Sezen, Gebze Institute of Technology E-mail: bsezen@gyte.edu.tr 


\begin{tabular}{|c|c|c|c|c|c|}
\hline DIMENSIONS & Long and healthy & \multicolumn{2}{|c|}{ Knowledge } & Decent standard of & Human Security \\
\hline INDICATORS & $\begin{array}{l}\text { Life expectancy at } \\
\text { birth }\end{array}$ & $\begin{array}{l}\text { Adult } \\
\text { Literacy } \\
\text { Rate }\end{array}$ & $\begin{array}{l}\text { Combined } \\
\text { Enrolment } \\
\text { Ratio }\end{array}$ & $\begin{array}{l}\text { Real GDP per capita } \\
\text { (PPP in US\$) }\end{array}$ & $\begin{array}{l}\text { Total } \\
\text { Unemployment Rate } \\
\text { (\% of labour force) }\end{array}$ \\
\hline $\begin{array}{l}\text { DIMENSION } \\
\text { INDICES }\end{array}$ & Longevity index & \multicolumn{2}{|c|}{$\begin{array}{l}\text { Educational Attainment } \\
\text { index }\end{array}$} & GDP index & Employment index \\
\hline \multicolumn{6}{|c|}{ Employment-adjusted Human Development Index (E-HDI) } \\
\hline
\end{tabular}

Figure 1: Employment-adjusted Human Development Index (E-HDI)

The basic requirements which are accepted as indicators of a decent life style include accessibility to certain goods and services. These fundamentals are acknowledged as essential criteria by the Basic Needs approach to development.

Although defined differently in different studies, Basic Needs has always included guaranteeing such basic fundamentals as access to safe food (adequate nutrition) and water, and universal provision of health and education services with shelter, clothing and nonmaterial needs such as employment, participation, protection and political liberty. If any of these needs are absent or in a critically short supply, the problem of underdevelopment comes onto the scene (Ghosh 1984; Hicks and Streeten 1979; ILO 1976; UNDP 1994).

All attempts to achieve the expectation of fundamental human needs and development arise from the premise that development should be concerned with the elimination of absolute deprivation as a first priority.

For centuries, the constitutional rhetoric and aspirations of every leader and public figure in the developing and developed world have set forth these basic conditions as a fundamental right. It should also be mentioned that the basic needs approach seems to be consistent with Amartya Sen (1983)'s notion of "entitlement" and "capabilities".

It should be emphasized that the Basic Needs approach to development, while not a strategy, simply prioritizes the order of development techniques. Simply stated, the objectives themselves, and not the methods of implementation to arrive at these objectives, are the more important focus of the approach. (Stewart, 1995).
The Basic Needs approach prescribes a clear set of goals which address planning purposes and policy makers. These goals set the improvement of the quality of life of the indiviual as their main focus. Therefore, health, education, employment and a commensurate income can be seen as a measure of achieving lifelong goals.

The search for an additional dimension for the composite index of socio-economic progress, namely the human development index (HDI), began in the early 1990's soon after it was introduced by the United Nations Development Programme. For it to be widely accepted and used, it is crucial that the HDI includes only the most important and a limited number of accessible variables for all nations, in order to keep it simple and manageable. The selection of these dimensions highly depends on how the society regards and defines an acceptable composite.

On the other hand, employment could be considered as a vector to raise personal income, which in turn secures the access of individuals to more goods and services. Therefore, it is our recommendation that employment or decent work should be regarded as one of the most important dimensions of human development. Hence, adding the employment dimension under the label of "human security" to the HDI will yield a more comprehensive measure of human development that captures more of people's needs for improving their way of life.

It is important to note that the employment data of many countries are readily available in contrast to variables from social, cultural and political dimensions. 
As shown in Figure 1, in addition to the three essential choices of leading a long and healthy life, acquiring knowledge, having access to resources needed for a decent standard of living; and having security needed for a sustainable life are included in the measure of the EHDI.

In today's world, employment can be recognized as a fundamental human right. It brings personal economic freedom. Additionally, providing and implementing strategies for meaningful and productive work for young generations is one of the main targets of the Millennium Development Goals. Thus, the capacity to develop and satisfy the job needs of its citizens must be among the major goals of every nation in the coming decades. Employment can be further considered a physical need. It constitutes the essential basis for peace, social justice, food security and human development. In this context, securing full employment levels can be considered one of the primary objectives of every nation. Moreover, rising employment levels is also beneficial in fostering economic growth and attaining sustainable development.

In short, employment could be regarded as one of the most critical indicators of human development. Economies functioning at a full employment level, and thus, high levels of gross domestic product (GDP) show radical improvements in terms of human development.

In other words, employment is positively related to the HDI. Its generation is particularly significant for economic growth and poverty reduction. Raising the employment level has been the major objective of the International Labor Organization since the early 1970s due to the fact that employment is not only considered an objective in itself but also a moderator to achieve various objectives, such as high levels of income, production and recognition. Therefore, one should argue that the employment-adjusted index has a great potential to make the HDI more operational.

\section{The Rationale of the Study}

The current HDI has a limited capacity to reflect the human condition and country rankings in an accurate way. In addition, the main critiques of the HDI suggest that it uses very few or perhaps the wrong indicators in measuring the human development levels of countries. This is mainly due to the fact that two of the current components used for calculating the $\mathrm{HDI}$ are not dynamic. It is true that the HDI value is developed to evolve with time; however, current HDI components fail to yield significantly perceiveable overall annual increases to affect the $\mathrm{HDI}$ value. This drawback lowers the sensitivity of the HDI towards annual changes.

Nonetheless, employment is a dynamic and fluctuating dimension which reflects the real life circumstances of the individual, similar to health, education and access to resources. It also shows considerable variation across countries.

The unemployment rate, although it has not yet been included in the sub-indices of the $\mathrm{HDI}$, is a strong indicator of social inclusion and quality-of-life through the efficient use of human resources (Taner et al. 2011). In addition, Panigrahi and Sivramkrishna (2002), Osberg and Sharpe (2003) and Cherchye et al. (2008) have presented their concerns with the problems in $\mathrm{HDI}$ rankings in their publications. Furthermore, Wolff et al. (2009) and Taner et al. (2010), in their analyses, have suggested, and substantiated with statistics, that countries have been misclassified by the HDI.

Hence, this paper empirically investigates whether the inclusion of employment as a criterion in the HDI would yield a different ranking of nations. It is further argued that the HDI could be appropriately modified by simply incorporating an employment dimension to the current index. This Employment-adjusted Human Development Index is denoted as the E-HDI. Due to the limited availability of data for other countries, the sample addresses the situation in only seventy-seven countries.

\section{Method}

Formerly, the HDI had been based on three subindices and four indicators: a longevity index (LEI), as measured by life expectancy at birth; an educational attainment index, as measured by a combination of adult literacy (two-thirds weighting) and combined (i.e. primary, secondary and tertiary) enrolment (one-third weighting) ratios (EI); and standard of living, as measured by GDP index -real GDP per capita measured by purchasing power parity in US\$- (GDPI). To calibrate the dimensions, the UNDP has assigned minimum and maximum values (goalposts) for each underlying subindex. Performance in each sub-index is then calculated and expressed as a value between 0-1. In the UNDP's approach, these three sub-indices are assigned equal weightings as follows:

$\mathrm{HDI}=(\mathrm{LEI}+\mathrm{EI}+\mathrm{GDPI}) / 3$ 
As indicated above, the HDI has included only a limited number of indicators to keep it simple and manageable. This simple HDI algorithm has been used for many years and calculated from regularly available data to produce a meaningful value that can be used to compare and rank countries across the world.

In the present approach, the four indices in the E-HDI represent a different set of indicators for assessing the aggregate level of human development with equal weighting in the following way:

$$
\begin{aligned}
& \mathrm{E}-\mathrm{HDI}=(\mathrm{LEI}+\mathrm{EI}+\mathrm{GDPI}+\mathrm{EMPI}) / 4, \text { or } \\
& \mathrm{E}-\mathrm{HDI}=(3 * \mathrm{HDI}+\mathrm{EMPI}) / 4
\end{aligned}
$$

where EMPI is the new included index, i.e. employment index. The unemployment rate is the starting point for the EMPI sub-index of the human security component. This, together with the risk of losing one's job, combined with being unable to find a new job quickly, is taken as a measure of what drives worker insecurity. The EMPI is likewise calculated via basic algebra for each country as shown below:

\section{EMPI = 1 - Total Unemployment Rate}

The proposed sub-index of EMPI covers all individuals of normal working age (above 15) including both the employed and the unemployed. In addition, the four subindices address conceptually different aspects of human development, which although correlated do not predetermine one another.

The equal weights allow easy comparison over time and across countries. The assessment of change in E-HDI over the years for all the countries is tabulated through using statistical tables. From E-HDI values, comparisons of achievements between countries at a given year or for a particular country for different periods can also be made. Like the HDI, the E-HDI captures both trends over time within countries and allows cross-country comparisons of the level of human development at particular points in time.

\section{Data}

In this study, a total of 77 highly developed, developed and developing economies are taken as the research sample; re-ranked and re-classified among themselves for the years between 2000 and 2007'. Scaled values of the sub-indices are shown in Table 1, 2 and 4-8.

The countries consisted mainly European Union (EU), OECD, Asian, and Central and South American countries. The limitation of the sample size was due to the lack of unemployment data for the rest of the countries for most of the years under study. Another limitation of the study is that statistical analysis has not been possible for the most recent data covered by the 2010 and 2011 Human Development Reports, since the UNDP has opted to change both education and income indicators and the method of calculating HDI in these reports.

\section{Descriptive Statistical Analysis}

This section presents the main findings of descriptive statistical analysis. In the first sub-section, the main trends for all countries in the sample are thoroughly reviewed, focusing on the HDI, E-HDI values and the rankings together with basic changes in these indicators throughout the period under investigation. Consequently, the analysis is detailed through dividing the sample of 77 countries into two main categories under the titles of OECD and developing countries. While the first category consists of the 30 countries presented at the top of the statistical tables, the remaining 47 countries are covered by the developing country category.

\subsection{General Evaluation}

The sample consists of 38 European, 16 Asian, 10 South American, 9 Central and North American, 2 Oceanian and 2 African countries. Of the 38 European countries, from the latest available data it is found that 22 are very highly employment-adjusted developed (EAD) where the absolute values of the E-HDI exceed a threshold level of $0.900,15$ are highly EAD (the values of the E-HDI are between 0.800 and 0.900 ) and only Macedonia is moderately EAD (the values of the E-HDI are between 0.700 and 0.800 ). Both of the Oceanian countries are very highly EAD. Of the 16 Asian countries, 7 are very highly employment-adjusted developed (EAD), 4 are highly EAD and 5 are moderately EAD. Of the 10 South American countries, 9 are highly EAD and only Bolivia is moderately EAD. Of the 9 Central and North American countries, 2 are very highly employment-adjusted

${ }^{1}$ The data for the HDI and its components is taken from UNDP's Human Development Reports from 2002 to 2009. 


\begin{tabular}{|c|c|c|c|c|c|c|c|c|c|c|}
\hline & LEI & GDPI & EMPI & EI & E-HDI & HDI & HDI-E-HDI & R(HDI) & R(E-HDI) & R(HDI-E-HDI) \\
\hline Iceland & 0,90 & 0,95 & 0,986 & 0,96 & 0,949 & 0,936 & $-0,013$ & 7 & 1 & 6 \\
\hline Norway & 0,89 & 0,95 & 0,966 & 0,98 & 0,9465 & 0,942 & $-0,0045$ & 1 & 2 & -1 \\
\hline Australia & 0,90 & 0,93 & 0,937 & 0,99 & 0,93925 & 0,939 & $-0,00025$ & 4 & 7 & -3 \\
\hline Canada & 0,90 & 0,94 & 0,932 & 0,98 & 0,938 & 0,94 & 0,002 & 3 & 8 & -5 \\
\hline Ireland & 0,86 & 0,95 & 0,957 & 0,96 & 0,93175 & 0,925 & $-0,00675$ & 16 & 13 & 3 \\
\hline Sweden & 0,91 & 0,92 & 0,953 & 0,99 & 0,94325 & 0,941 & $-0,00225$ & 2 & 5 & -3 \\
\hline Switzerland & 0,90 & 0,94 & 0,98 & 0,94 & 0,94 & 0,928 & $-0,012$ & 11 & 6 & 5 \\
\hline Japan & 0,93 & 0,93 & 0,953 & 0,93 & 0,93575 & 0,933 & $-0,00275$ & 9 & 11 & -2 \\
\hline Netherlands & 0,89 & 0,93 & 0,974 & 0,99 & 0,946 & 0,935 & $-0,011$ & 8 & 3 & 5 \\
\hline France & 0,89 & 0,92 & 0,905 & 0,99 & 0,92625 & 0,928 & 0,00175 & 11 & 16 & -5 \\
\hline Finland & 0,88 & 0,92 & 0,902 & 0,99 & 0,923 & 0,93 & 0,007 & 10 & 18 & -8 \\
\hline United States & 0,87 & 0,97 & 0,96 & 0,98 & 0,945 & 0,939 & $-0,006$ & 4 & 4 & 0 \\
\hline Spain & 0,89 & 0,88 & 0,859 & 0,97 & 0,89975 & 0,913 & 0,01325 & 20 & 25 & -5 \\
\hline Denmark & 0,85 & 0,94 & 0,953 & 0,98 & 0,93075 & 0,926 & $-0,00475$ & 14 & 15 & -1 \\
\hline Austria & 0,89 & 0,93 & 0,953 & 0,96 & 0,93325 & 0,926 & $-0,00725$ & 14 & 12 & 2 \\
\hline United Kingdom & 0,88 & 0,91 & 0,945 & 0,99 & 0,93125 & 0,928 & $-0,00325$ & 11 & 14 & -3 \\
\hline Belgium & 0,89 & 0,94 & 0,93 & 0,99 & 0,9375 & 0,939 & 0,0015 & 4 & 9 & -5 \\
\hline Luxembourg & 0,87 & 1 & 0,974 & 0,9 & 0,936 & 0,925 & $-0,011$ & 16 & 10 & 6 \\
\hline New Zealand & 0,88 & 0,88 & 0,94 & 0,99 & 0,9225 & 0,917 & $-0,0055$ & 19 & 19 & 0 \\
\hline Italy & 0,89 & 0,91 & 0,893 & 0,94 & 0,90825 & 0,913 & 0,00475 & 20 & 20 & 0 \\
\hline Germany & 0,88 & 0,92 & 0,925 & 0,97 & 0,92375 & 0,925 & 0,00125 & 16 & 17 & -1 \\
\hline Greece & 0,89 & 0,85 & 0,886 & 0,92 & 0,8865 & 0,896 & 0,0095 & 22 & 28 & -6 \\
\hline Republic of Korea & 0,83 & 0,86 & 0,959 & 0,95 & 0,89975 & 0,888 & $-0,01175$ & 24 & 25 & -1 \\
\hline Portugal & 0,84 & 0,86 & 0,96 & 0,94 & 0,9 & 0,885 & $-0,015$ & 26 & 24 & 2 \\
\hline Czech Republic & 0,83 & 0,82 & 0,911 & 0,89 & 0,86275 & 0,885 & 0,02225 & 26 & 29 & -3 \\
\hline Hungary & 0,77 & 0,8 & 0,935 & 0,93 & 0,85875 & 0,883 & 0,02425 & 29 & 29 & 0 \\
\hline Poland & 0,81 & 0,75 & 0,839 & 0,94 & 0,83475 & 0,88 & 0,04525 & 32 & 39 & -7 \\
\hline Slovakia & 0,80 & 0,79 & 0,812 & 0,91 & 0,828 & 0,882 & 0,054 & 31 & 42 & -11 \\
\hline Mexico & 0,79 & 0,75 & 0,978 & 0,84 & 0,8395 & 0,8 & $-0,0395$ & 44 & 35 & 9 \\
\hline Turkey & 0,75 & 0,71 & 0,936 & 0,77 & 0,7915 & 0,742 & $-0,0495$ & 61 & 52 & 9 \\
\hline Estonia & 0,76 & 0,77 & 0,864 & 0,95 & 0,836 & 0,826 & $-0,01$ & 39 & 38 & 1 \\
\hline Georgia & 0,8 & 0,55 & 0,892 & 0,89 & 0,783 & 0,748 & $-0,035$ & 58 & 58 & 0 \\
\hline Hong Kong & 0,91 & 0,92 & 0,951 & 0,83 & 0,90275 & 0,888 & $-0,01475$ & 24 & 22 & 2 \\
\hline Israel & 0,9 & 0,89 & 0,912 & 0,91 & 0,903 & 0,896 & $-0,007$ & 22 & 21 & 1 \\
\hline Latvia & 0,76 & 0,71 & 0,856 & 0,93 & 0,814 & 0,8 & $-0,014$ & 44 & 46 & -2 \\
\hline Lithuania & 0,78 & 0,71 & 0,836 & 0,93 & 0,814 & 0,808 & $-0,006$ & 42 & 46 & -4 \\
\hline Malaysia & 0,79 & 0,75 & 0,97 & 0,8 & 0,8275 & 0,782 & $-0,0455$ & 49 & 43 & 6 \\
\hline Argentina & 0,81 & 0,8 & 0,85 & 0,92 & 0,845 & 0,844 & $-0,001$ & 34 & 34 & 0 \\
\hline Bulgaria & 0,76 & \begin{tabular}{|l|}
0,68 \\
\end{tabular} & 0,837 & 0,9 & 0,79425 & 0,779 & $-0,01525$ & 51 & 51 & 0 \\
\hline Chile & 0,84 & 0,76 & 0,917 & 0,9 & 0,85425 & 0,831 & $-0,02325$ & 37 & 33 & 4 \\
\hline Croatia & 0,81 & 0,73 & 0,839 & 0,88 & 0,81475 & 0,809 & $-0,00575$ & 41 & 45 & -4 \\
\hline China & 0,76 & 0,61 & 0,969 & 0,8 & 0,78475 & 0,726 & $-0,05875$ & 67 & 55 & 12 \\
\hline Russia & 0,68 & 0,74 & 0,902 & 0,92 & 0,8105 & 0,781 & $-0,0295$ & 50 & 49 & 1 \\
\hline Belarus & 0,73 & 0,72 & 0,979 & 0,92 & 0,83725 & 0,788 & $-0,04925$ & 47 & 37 & 10 \\
\hline Cuba & 0,85 & 0,64 & 0,946 & 0,9 & 0,834 & 0,795 & $-0,039$ & 46 & 40 & 6 \\
\hline Ecuador & 0,75 & 0,58 & 0,91 & 0,87 & 0,7775 & 0,732 & $-0,0455$ & 64 & 59 & 5 \\
\hline Panama & 0,82 & 0,68 & 0,865 & 0,86 & 0,80625 & 0,787 & $-0,01925$ & 48 & 50 & -2 \\
\hline Romania & 0,75 & 0,69 & 0,929 & 0,88 & 0,81225 & 0,775 & $-0,03725$ & 52 & 48 & 4 \\
\hline
\end{tabular}




\begin{tabular}{|c|c|c|c|c|c|c|c|c|c|c|}
\hline & LEI & GDPI & EMPI & EI & E-HDI & HDI & HDI-E-HDI & R(HDI) & R(E-HDI) & R(HDI-E-HDI) \\
\hline Trinidad & 0,82 & 0,75 & 0,878 & 0,84 & 0,822 & 0,805 & $-0,017$ & 43 & 44 & -1 \\
\hline Ukraine & 0,72 & 0,61 & 0,884 & 0,92 & 0,7835 & 0,748 & $-0,0355$ & 58 & 56 & 2 \\
\hline Slovakia & 0,8 & 0,79 & 0,814 & 0,91 & 0,8285 & 0,835 & 0,0065 & 35 & 41 & -6 \\
\hline Paraguay & 0,75 & 0,63 & 0,924 & 0,83 & 0,7835 & 0,74 & $-0,0435$ & 62 & 56 & 6 \\
\hline Uruguay & 0,82 & 0,75 & 0,864 & 0,92 & 0,8385 & 0,831 & $-0,0075$ & 36 & 36 & 0 \\
\hline South Africa & 0,45 & 0,76 & 0,746 & 0,88 & 0,709 & 0,695 & $-0,014$ & 71 & 68 & 3 \\
\hline Malta & 0,88 & 0,86 & 0,933 & 0,88 & 0,88825 & 0,875 & $-0,01325$ & 33 & 27 & 6 \\
\hline Moldova & 0,69 & 0,51 & 0,927 & 0,9 & 0,75675 & 0,701 & $-0,05575$ & 70 & 64 & 6 \\
\hline Kyrgyzstan & 0,71 & 0,55 & 0,925 & 0,87 & 0,76375 & 0,712 & $-0,05175$ & 68 & 61 & 7 \\
\hline Cyprus & 0,88 & 0,89 & 0,951 & 0,88 & 0,90025 & 0,883 & $-0,01725$ & 29 & 23 & 6 \\
\hline Albania & 0,8 & 0,59 & 0,832 & 0,8 & 0,7555 & 0,733 & $-0,0225$ & 63 & 65 & -2 \\
\hline Bolivia & 0,62 & 0,53 & 0,925 & 0,8 & 0,71875 & 0,653 & $-0,06575$ & 74 & 67 & 7 \\
\hline Kuwait & 0,85 & 0,84 & 0,992 & 0,74 & 0,8555 & 0,813 & $-0,0425$ & 40 & 32 & 8 \\
\hline Nicaragua & 0,72 & 0,53 & $\mathrm{n} / \mathrm{a}$ & 0,65 & $\mathrm{n} / \mathrm{a}$ & 0,635 & $\mathrm{n} / \mathrm{a}$ & 77 & $\mathrm{n} / \mathrm{a}$ & $\mathrm{n} / \mathrm{a}$ \\
\hline Macedonia & 0,8 & 0,66 & $\mathrm{n} / \mathrm{a}$ & 0,86 & $\mathrm{n} / \mathrm{a}$ & 0,772 & $\mathrm{n} / \mathrm{a}$ & 54 & $\mathrm{n} / \mathrm{a}$ & $\mathrm{n} / \mathrm{a}$ \\
\hline Singapore & 0,88 & 0,91 & $\mathrm{n} / \mathrm{a}$ & 0,87 & $\mathrm{n} / \mathrm{a}$ & 0,885 & $\mathrm{n} / \mathrm{a}$ & 26 & $\mathrm{n} / \mathrm{a}$ & $\mathrm{n} / \mathrm{a}$ \\
\hline Tajikistan & 0,71 & 0,41 & 0,827 & 0,88 & 0,70675 & 0,667 & $-0,03975$ & 73 & 70 & 3 \\
\hline Uzbekistan & 0,73 & 0,53 & 0,864 & 0,91 & 0,7585 & 0,727 & $-0,0315$ & 65 & 63 & 2 \\
\hline Peru & 0,73 & 0,65 & $\mathrm{n} / \mathrm{a}$ & 0,87 & $\mathrm{n} / \mathrm{a}$ & 0,747 & $\mathrm{n} / \mathrm{a}$ & 60 & $\mathrm{n} / \mathrm{a}$ & $\mathrm{n} / \mathrm{a}$ \\
\hline Colombia & 0,77 & 0,69 & 0,834 & 0,85 & 0,786 & 0,772 & $-0,014$ & 53 & 54 & -1 \\
\hline Philippines & 0,74 & 0,61 & 0,888 & 0,91 & 0,787 & 0,754 & $-0,033$ & 56 & 53 & 3 \\
\hline Indonesia & 0,69 & 0,57 & 0,939 & 0,79 & 0,74725 & 0,684 & $-0,06325$ & 72 & 66 & 6 \\
\hline Egypt & 0,7 & 0,6 & 0,91 & 0,62 & 0,7075 & 0,642 & $-0,0655$ & 75 & 69 & 6 \\
\hline El Salvador & 0,75 & 0,64 & 0,93 & 0,74 & 0,765 & 0,706 & $-0,059$ & 69 & 60 & 9 \\
\hline Honduras & 0,68 & 0,53 & $\mathrm{n} / \mathrm{a}$ & 0,7 & $\mathrm{n} / \mathrm{a}$ & 0,638 & $\mathrm{n} / \mathrm{a}$ & 76 & $\mathrm{n} / \mathrm{a}$ & $\mathrm{n} / \mathrm{a}$ \\
\hline Dominican Republic & 0,7 & 0,68 & 0,861 & 0,8 & 0,76025 & 0,727 & $-0,03325$ & 65 & 62 & 3 \\
\hline Bahrain & 0,81 & 0,84 & 0,939 & 0,85 & 0,85975 & 0,831 & $-0,02875$ & 36 & 30 & 6 \\
\hline Kazakhstan & 0,66 & 0,68 & $\mathrm{n} / \mathrm{a}$ & 0,91 & $\mathrm{n} / \mathrm{a}$ & 0,75 & $\mathrm{n} / \mathrm{a}$ & 57 & $\mathrm{n} / \mathrm{a}$ & $\mathrm{n} / \mathrm{a}$ \\
\hline Brazil & 0,71 & 0,72 & $\mathrm{n} / \mathrm{a}$ & 0,83 & $\mathrm{n} / \mathrm{a}$ & 0,757 & $\mathrm{n} / \mathrm{a}$ & 55 & $\mathrm{n} / \mathrm{a}$ & $\mathrm{n} / \mathrm{a}$ \\
\hline
\end{tabular}

EMPI, E-HDI, R(E-HDI): Authors' own calculations

$\mathrm{HDI}-\mathrm{E}-\mathrm{HDI}=$ Numerical difference between the two indices

$\mathrm{R}(\mathrm{HDI})=$ Rank of a country in terms of its $\mathrm{HDI}$

$\mathrm{R}(\mathrm{E}-\mathrm{HDI})=$ Rank of a country in terms of its E-HDI

$\mathrm{R}(\mathrm{HDI}-\mathrm{E}-\mathrm{HDI})=$ Difference between the $\mathrm{HDI}$ ranking and $\mathrm{E}-\mathrm{HDI}$ rank of a country

Source: UNDP (2002); ILO

Table 1: Employment- adjusted Human Development Index (E-HDI): 2000 


\begin{tabular}{|c|c|c|c|c|c|c|c|c|c|c|}
\hline & LEI & GDPI & EMPI & EI & E-HDI & HDI & HDI-E-HDI & $\mathrm{R}(\mathrm{HDI})$ & R(E-HDI) & R(HDI-E-HDI) \\
\hline Iceland & 0,95 & 0,981 & 0,97 & 0,98 & \begin{tabular}{|l|}
0,96925 \\
\end{tabular} & 0,969 & $-0,00025$ & 3 & 2 & 1 \\
\hline Norway & 0,93 & 1 & 0,974 & 0,989 & 0,972 & 0,971 & $-0,001$ & 1 & 1 & 0 \\
\hline Australia & 0,94 & 0,977 & 0,958 & 0,993 & 0,967 & 0,97 & 0,003 & 2 & 3 & -1 \\
\hline Canada & 0,93 & 0,982 & 0,939 & 0,991 & 0,95975 & 0,966 & 0,00625 & 4 & 7 & -3 \\
\hline Ireland & 0,91 & 1 & 0,94 & 0,985 & 0,959 & 0,965 & 0,006 & 5 & 8 & -3 \\
\hline Sweden & 0,93 & 0,986 & 0,938 & 0,974 & 0,957 & 0,963 & 0,006 & 7 & 11 & -4 \\
\hline Switzerland & 0,95 & 1 & 0,966 & 0,936 & \begin{tabular}{|l|}
0,96175 \\
\end{tabular} & 0,96 & $-0,00175$ & 9 & 5 & 4 \\
\hline Japan & 0,96 & 0,971 & 0,96 & 0,949 & 0,96025 & 0,96 & $-0,00025$ & 9 & 6 & 3 \\
\hline Netherlands & 0,91 & 0,994 & 0,972 & 0,985 & \begin{tabular}{|l|}
0,96625 \\
\end{tabular} & 0,964 & $-0,00225$ & 6 & 4 & 2 \\
\hline France & 0,93 & 0,971 & 0,926 & 0,978 & 0,952 & 0,961 & 0,009 & 8 & 15 & -7 \\
\hline Finland & 0,91 & 0,975 & 0,936 & 0,993 & 0,953 & 0,959 & 0,006 & 12 & 13 & -1 \\
\hline United States & 0,90 & 1 & 0,942 & 0,968 & 0,953 & 0,956 & 0,003 & 13 & 13 & 0 \\
\hline Spain & 0,93 & 0,96 & 0,887 & 0,975 & 0,93775 & 0,955 & 0,01725 & 14 & 24 & -10 \\
\hline Denmark & 0,89 & 0,983 & 0,967 & 0,993 & 0,9575 & 0,955 & $-0,0025$ & 14 & 9 & 5 \\
\hline Austria & 0,92 & 0,989 & 0,962 & 0,962 & 0,957 & 0,955 & $-0,002$ & 14 & 11 & 3 \\
\hline United Kingdom & 0,91 & 0,978 & 0,944 & 0,957 & 0,94625 & 0,947 & 0,00075 & 20 & 21 & -1 \\
\hline Belgium & 0,91 & 0,977 & 0,93 & 0,974 & \begin{tabular}{|l|}
0,94725 \\
\end{tabular} & 0,953 & 0,00575 & 17 & 19 & -2 \\
\hline Luxembourg & 0,91 & 1 & 0,949 & 0,975 & 0,9575 & 0,96 & 0,0025 & 9 & 9 & 0 \\
\hline New Zealand & 0,92 & 0,936 & 0,959 & 0,993 & \begin{tabular}{|l|}
0,95175 \\
\end{tabular} & 0,95 & $-0,00175$ & 19 & 16 & 3 \\
\hline Italy & 0,94 & 0,954 & 0,933 & 0,965 & 0,94675 & 0,951 & 0,00425 & 18 & 20 & -2 \\
\hline Germany & 0,91 & 0,975 & 0,925 & 0,954 & 0,94175 & 0,947 & 0,00525 & 20 & 23 & -3 \\
\hline Greece & 0,90 & 0,944 & 0,923 & 0,981 & 0,9375 & 0,942 & 0,0045 & 24 & 25 & -1 \\
\hline Republic of Korea & 0,90 & 0,92 & 0,968 & 0,988 & 0,945 & 0,937 & $-0,008$ & 25 & 22 & 3 \\
\hline Portugal & 0,89 & 0,906 & 0,924 & 0,929 & 0,913 & 0,909 & $-0,004$ & 29 & 28 & 1 \\
\hline Czech Republic & 0,86 & 0,916 & 0,956 & 0,938 & 0,9165 & 0,903 & $-0,0135$ & 30 & 27 & 3 \\
\hline Hungary & 0,81 & 0,874 & 0,922 & 0,96 & 0,89025 & 0,879 & $-0,01125$ & 37 & 36 & 1 \\
\hline Poland & 0,84 & 0,847 & 0,929 & 0,952 & 0,8925 & 0,88 & $-0,0125$ & 34 & 33 & 1 \\
\hline Slovakia & 0,83 & 0,885 & 0,905 & 0,928 & 0,88625 & 0,88 & $-0,00625$ & 34 & 37 & -3 \\
\hline Mexico & 0,85 & 0,826 & 0,96 & 0,886 & 0,8805 & 0,854 & $-0,0265$ & 45 & 40 & 5 \\
\hline Turkey & 0,78 & 0,812 & 0,906 & 0,828 & 0,83125 & 0,806 & $-0,02525$ & 57 & 54 & 3 \\
\hline Estonia & 0,799 & 0,887 & 0,953 & 0,964 & 0,90075 & 0,883 & $-0,01775$ & 33 & 31 & 2 \\
\hline Georgia & 0,777 & 0,641 & 0,867 & 0,916 & 0,80025 & 0,778 & $-0,02225$ & 62 & 60 & 2 \\
\hline Hong Kong & 0,953 & 1 & 0,96 & 0,879 & 0,948 & 0,944 & $-0,004$ & 22 & 18 & 4 \\
\hline Israel & 0,928 & 0,93 & 0,927 & 0,947 & 0,933 & 0,935 & 0,002 & 26 & 26 & 0 \\
\hline Latvia & 0,788 & 0,851 & 0,94 & 0,961 & 0,885 & 0,866 & $-0,019$ & 41 & 38 & 3 \\
\hline Lithuania & 0,78 & 0,863 & 0,957 & 0,968 & 0,892 & 0,87 & $-0,022$ & 40 & 34 & 6 \\
\hline Malaysia & 0,819 & 0,819 & 0,968 & 0,851 & 0,86425 & 0,829 & $-0,03525$ & 50 & 44 & 6 \\
\hline Argentina & 0,831 & 0,828 & $\mathrm{n} / \mathrm{a}$ & 0,947 & $\mathrm{n} / \mathrm{a}$ & 0,866 & $\mathrm{n} / \mathrm{a}$ & 41 & $\mathrm{n} / \mathrm{a}$ & $\mathrm{n} / \mathrm{a}$ \\
\hline Bulgaria & 0,802 & 0,788 & 0,931 & 0,93 & 0,86275 & 0,84 & $-0,02275$ & 46 & 47 & -1 \\
\hline Chile & 0,891 & 0,823 & 0,929 & 0,919 & 0,8905 & 0,878 & $-0,0125$ & 38 & 35 & 3 \\
\hline Croatia & 0,85 & 0,847 & 0,904 & 0,916 & 0,87925 & 0,871 & $-0,00825$ & 39 & 41 & -2 \\
\hline China & 0,799 & 0,665 & 0,96 & 0,851 & 0,81875 & 0,772 & $-0,04675$ & 64 & 58 & 6 \\
\hline Russia & 0,686 & 0,833 & 0,939 & 0,933 & 0,84775 & 0,817 & $-0,03075$ & 53 & 49 & 4 \\
\hline Belarus & 0,733 & 0,782 & 0,99 & 0,961 & 0,8665 & 0,826 & $-0,0405$ & 51 & 43 & 8 \\
\hline Cuba & 0,891 & 0,706 & 0,982 & 0,993 & 0,893 & 0,863 & $-0,03$ & 44 & 32 & 12 \\
\hline Ecuador & 0,833 & 0,719 & 0,939 & 0,866 & 0,83925 & 0,806 & $-0,03325$ & 57 & 50 & 7 \\
\hline Panama & 0,842 & 0,79 & 0,932 & 0,888 & 0,863 & 0,84 & $-0,023$ & 46 & 46 & 0 \\
\hline Romania & 0,792 & 0,804 & 0,936 & 0,915 & 0,86175 & 0,837 & $-0,02475$ & 48 & 48 & 0 \\
\hline
\end{tabular}




\begin{tabular}{|c|c|c|c|c|c|c|c|c|c|c|}
\hline & LEI & GDPI & EMPI & EI & E-HDI & HDI & HDI-E-HDI & R(HDI) & R(E-HDI) & R(HDI-E-HDI) \\
\hline Trinidad & 0,737 & 0,911 & 0,945 & 0,861 & 0,8635 & 0,837 & $-0,0265$ & 48 & 45 & 3 \\
\hline Ukraine & 0,72 & 0,707 & 0,936 & 0,96 & 0,83075 & 0,796 & $-0,03475$ & 61 & 55 & 6 \\
\hline Slovakia & 0,827 & 0,885 & 0,89 & 0,928 & 0,8825 & 0,88 & $-0,0025$ & 34 & 39 & -5 \\
\hline Paraguay & 0,778 & 0,633 & 0,944 & 0,871 & 0,8065 & 0,761 & $-0,0455$ & 65 & 59 & 6 \\
\hline Uruguay & 0,852 & 0,788 & 0,908 & 0,955 & 0,87575 & 0,865 & $-0,01075$ & 43 & 42 & 1 \\
\hline South Africa & 0,442 & 0,765 & 0,77 & 0,843 & 0,705 & 0,683 & $-0,022$ & 77 & 74 & 3 \\
\hline Malta & 0,91 & 0,908 & 0,936 & 0,887 & 0,91025 & 0,902 & $-0,00825$ & 31 & 29 & 2 \\
\hline Moldova & 0,722 & 0,541 & 0,96 & 0,899 & 0,7805 & 0,72 & $-0,0605$ & 71 & 66 & 5 \\
\hline Kyrgyzstan & 0,71 & 0,5 & 0,927 & 0,918 & 0,76375 & 0,71 & $-0,05375$ & 72 & 69 & 3 \\
\hline Cyprus & 0,91 & 0,92 & 0,961 & 0,91 & 0,82275 & 0,914 & 0,09125 & 28 & 57 & -29 \\
\hline Albania & 0,858 & 0,71 & $\mathrm{n} / \mathrm{a}$ & 0,886 & $\mathrm{n} / \mathrm{a}$ & 0,818 & $\mathrm{n} / \mathrm{a}$ & 52 & $\mathrm{n} / \mathrm{a}$ & $\mathrm{n} / \mathrm{a}$ \\
\hline Bolivia & 0,673 & 0,624 & 0,948 & 0,892 & 0,78425 & 0,729 & $-0,05525$ & 70 & 65 & 5 \\
\hline Kuwait & 0,875 & 1 & $\mathrm{n} / \mathrm{a}$ & 0,872 & $\mathrm{n} / \mathrm{a}$ & 0,916 & $\mathrm{n} / \mathrm{a}$ & 27 & $\mathrm{n} / \mathrm{a}$ & $\mathrm{n} / \mathrm{a}$ \\
\hline Nicaragua & 0,795 & 0,542 & 0,951 & 0,76 & 0,762 & 0,699 & $-0,063$ & 75 & 70 & 5 \\
\hline Macedonia & 0,819 & 0,753 & 0,651 & 0,88 & 0,77575 & 0,817 & 0,04125 & 53 & 68 & -15 \\
\hline Singapore & 0,92 & 1 & 0,96 & 0,913 & 0,94825 & 0,944 & $-0,00425$ & 22 & 17 & 5 \\
\hline Tajikistan & 0,691 & 0,478 & 0,799 & 0,896 & 0,716 & 0,688 & $-0,028$ & 76 & 73 & 3 \\
\hline Uzbekistan & 0,711 & 0,532 & 0,908 & 0,888 & 0,75975 & 0,71 & $-0,04975$ & 72 & 71 & 1 \\
\hline Peru & 0,8 & 0,728 & 0,933 & 0,891 & 0,838 & 0,806 & $-0,032$ & 57 & 52 & 5 \\
\hline Colombia & 0,795 & 0,743 & 0,891 & 0,881 & 0,8275 & 0,807 & $-0,0205$ & 56 & 56 & 0 \\
\hline Philippines & 0,777 & 0,589 & 0,927 & 0,888 & 0,79525 & 0,751 & $-0,04425$ & 66 & 61 & 5 \\
\hline Indonesia & 0,758 & 0,603 & 0,909 & 0,84 & 0,7775 & 0,734 & $-0,0435$ & 68 & 67 & 1 \\
\hline Egypt & 0,749 & 0,664 & 0,911 & 0,697 & 0,75525 & 0,703 & $-0,05225$ & 74 & 72 & 2 \\
\hline El Salvador & 0,771 & 0,678 & 0,936 & 0,794 & 0,79475 & 0,747 & $-0,04775$ & 67 & 62 & 5 \\
\hline Honduras & 0,783 & 0,607 & 0,971 & 0,806 & 0,79175 & 0,732 & $-0,05975$ & 69 & 64 & 5 \\
\hline Dominican Republic & 0,79 & 0,702 & 0,844 & 0,839 & 0,79375 & 0,777 & $-0,01675$ & 63 & 63 & 0 \\
\hline Bahrain & 0,843 & 0,95 & 0,932 & 0,893 & 0,9045 & 0,895 & $-0,0095$ & 32 & 30 & 2 \\
\hline Kazakhstan & 0,666 & 0,782 & 0,927 & 0,965 & 0,835 & 0,804 & $-0,031$ & 60 & 53 & 7 \\
\hline Brazil & 0,787 & 0,761 & 0,918 & 0,891 & 0,83925 & 0,813 & $-0,02625$ & 55 & 51 & 4 \\
\hline
\end{tabular}

EMPI, E-HDI, R(E-HDI): Authors' own calculations.

Source: UNDP (2009); ILO

Table 2: Employment-adjusted Human Development Index (E-HDI): 2007 


\begin{tabular}{|c|c|c|c|c|c|c|c|c|}
\hline & \begin{tabular}{|c|} 
HDI \\
GROWTH \\
RATE \\
2000-2007 \\
(\% change) \\
\end{tabular} & $\begin{array}{c}\text { R-HDI } \\
2000 \\
\end{array}$ & R-HDI 2007 & $\begin{array}{c}\text { CHANGE } \\
\text { IN HDI } \\
\text { RANK } \\
\text { 2000-2007 } \\
\end{array}$ & $\begin{array}{c}\text { E-HDI } \\
\text { GROWTH } \\
\text { RATE } \\
2000-2007 \\
\text { (\% change) } \\
\end{array}$ & $\begin{array}{c}\text { R-E-HDI } \\
2000 \\
\end{array}$ & $\begin{array}{c}\text { R-E-HDI } \\
2007 \\
\end{array}$ & $\begin{array}{c}\text { CHANGE } \\
\text { IN E-HDI } \\
\text { RANK } \\
2000- \\
2007 \\
\end{array}$ \\
\hline Iceland & 3,526 & 7 & 3 & 4 & 2,134 & 1 & 2 & -1 \\
\hline Norway & 3,079 & 1 & 1 & 0 & 2,694 & 2 & 1 & 1 \\
\hline Australia & 3,301 & 4 & 2 & 2 & 2,954 & 7 & 3 & 4 \\
\hline Canada & 2,766 & 3 & 4 & -1 & 2,319 & 8 & 7 & 1 \\
\hline Ireland & 4,324 & 16 & 5 & 11 & 2,925 & 13 & 8 & 5 \\
\hline Sweden & 2,338 & 2 & 7 & -5 & 1,458 & 5 & 11 & -6 \\
\hline Switzerland & 3,448 & 11 & 9 & 2 & 2,314 & 6 & 5 & 1 \\
\hline Japan & 2,894 & 9 & 9 & 0 & 2,618 & 11 & 6 & 5 \\
\hline Netherlands & 3,102 & 8 & 6 & 2 & 2,141 & 3 & 4 & -1 \\
\hline France & 3,556 & 11 & 8 & 3 & 2,780 & 16 & 15 & 1 \\
\hline Finland & 3,118 & 10 & 12 & -2 & 3,250 & 18 & 13 & 5 \\
\hline United States & 1,810 & 4 & 13 & -9 & 0,847 & 4 & 13 & -9 \\
\hline Spain & 4,600 & 20 & 14 & 6 & 4,223 & 25 & 24 & 1 \\
\hline Denmark & 3,132 & 14 & 14 & 0 & 2,874 & 15 & 9 & 6 \\
\hline Austria & 3,132 & 14 & 14 & 0 & 2,545 & 12 & 11 & 1 \\
\hline United Kingdom & 2,047 & 11 & 20 & -9 & 1,611 & 14 & 21 & -7 \\
\hline Belgium & 1,491 & 4 & 17 & -13 & 1,040 & 9 & 19 & -10 \\
\hline Luxembourg & 3,784 & 16 & 9 & 7 & 2,297 & 10 & 9 & 1 \\
\hline New Zealand & 3,599 & 19 & 19 & 0 & 3,171 & 19 & 16 & 3 \\
\hline Italy & 4,162 & 20 & 18 & 2 & 4,239 & 20 & 20 & 0 \\
\hline Germany & 2,378 & 16 & 20 & -4 & 1,949 & 17 & 23 & -6 \\
\hline Greece & 5,134 & 22 & 24 & -2 & 5,753 & 28 & 25 & 3 \\
\hline Republic of Korea & 5,518 & 24 & 25 & -1 & 5,029 & 25 & 22 & 3 \\
\hline Portugal & 2,712 & 26 & 29 & -3 & 1,444 & 24 & 28 & -4 \\
\hline Czech Republic & 2,034 & 26 & 30 & -4 & 6,230 & 29 & 27 & 2 \\
\hline Hungary & $-0,453$ & 29 & 37 & -8 & 3,668 & 29 & 36 & -7 \\
\hline Poland & 0,000 & 32 & 34 & -2 & 6,918 & 39 & 33 & 6 \\
\hline Slovakia & $-0,227$ & 31 & 34 & -3 & 7,035 & 42 & 37 & 5 \\
\hline Mexico & 6,750 & 44 & 45 & -1 & 4,884 & 35 & 40 & -5 \\
\hline Turkey & 8,625 & 61 & 57 & 4 & 5,022 & 52 & 54 & -2 \\
\hline Estonia & 6,901 & 39 & 33 & 6 & 7,745 & 38 & 31 & 7 \\
\hline Georgia & 4,011 & 58 & 62 & -4 & 2,203 & 58 & 60 & -2 \\
\hline Hong Kong & 6,306 & 24 & 22 & 2 & 5,012 & 22 & 18 & 4 \\
\hline Israel & 4,353 & 22 & 26 & -4 & 3,322 & 21 & 26 & -5 \\
\hline Latvia & 8,250 & 44 & 41 & 3 & 8,722 & 46 & 38 & 8 \\
\hline Lithuania & 7,673 & 42 & 40 & 2 & 9,582 & 46 & 34 & 12 \\
\hline Malaysia & 6,010 & 49 & 50 & -1 & 4,441 & 43 & 44 & -1 \\
\hline Argentina & 2,607 & 34 & 41 & -7 & $3,609 * *$ & 34 & $37^{* * * * *}$ & $-3 * * * * * *$ \\
\hline Bulgaria & 7,831 & 51 & 46 & 5 & 8,624 & 51 & 47 & 4 \\
\hline Chile & 5,656 & 37 & 38 & -1 & 4,243 & 33 & 35 & -2 \\
\hline Croatia & 7,664 & 41 & 39 & 2 & 7,917 & 45 & 41 & 4 \\
\hline China & 6,336 & 67 & 64 & 3 & 4,333 & 55 & 58 & -3 \\
\hline Russia & 4,609 & 50 & 53 & -3 & 4,596 & 49 & 49 & 0 \\
\hline Belarus & 4,822 & 47 & 51 & -4 & 3,494 & 37 & 43 & -6 \\
\hline Cuba & 8,553 & 46 & 44 & 2 & 7,074 & 40 & 32 & 8 \\
\hline
\end{tabular}




\begin{tabular}{|c|c|c|c|c|c|c|c|c|}
\hline & $\begin{array}{c}\text { HDI } \\
\text { GROWTH } \\
\text { RATE } \\
\text { 2000-2007 } \\
\text { (\% change) }\end{array}$ & $\begin{array}{l}\text { R-HDI } \\
2000\end{array}$ & R-HDI 2007 & $\begin{array}{c}\text { CHANGE } \\
\text { IN HDI } \\
\text { RANK } \\
\text { 2000-2007 }\end{array}$ & $\begin{array}{c}\text { E-HDI } \\
\text { GROWTH } \\
\text { RATE } \\
\text { 2000-2007 } \\
\text { (\% change) }\end{array}$ & $\begin{array}{c}\text { R-E-HDI } \\
2000\end{array}$ & $\begin{array}{c}\text { R-E-HDI } \\
2007\end{array}$ & $\begin{array}{c}\text { CHANGE } \\
\text { IN E-HDI } \\
\text { RANK } \\
2000- \\
2007 \\
\end{array}$ \\
\hline Ecuador & 10,109 & 64 & 57 & 7 & 7,942 & 59 & 50 & 9 \\
\hline Panama & 6,734 & 48 & 46 & 2 & 7,039 & 50 & 46 & 4 \\
\hline Romania & 8,000 & 52 & 48 & 4 & 6,094 & 48 & 48 & 0 \\
\hline Trinidad & 3,975 & 43 & 48 & -5 & 5,049 & 44 & 45 & -1 \\
\hline Ukraine & 6,417 & 58 & 61 & -3 & 6,031 & 56 & 55 & 1 \\
\hline Slovakia & 5,389 & 35 & 34 & 1 & 6,518 & 41 & 39 & 2 \\
\hline Paraguay & 2,838 & 62 & 65 & -3 & 2,936 & 56 & 59 & -3 \\
\hline Uruguay & 4,091 & 36 & 43 & -7 & 4,442 & 36 & 42 & -6 \\
\hline South Africa & $-1,727$ & 71 & 77 & -6 & $-0,564$ & 68 & 74 & -6 \\
\hline Malta & 3,086 & 33 & 31 & 2 & 2,477 & 27 & 29 & -2 \\
\hline Moldova & 2,710 & 70 & 71 & -1 & 3,138 & 64 & 66 & -2 \\
\hline Kyrgyzstan & $-0,281$ & 68 & 72 & -4 & 0,000 & 61 & 69 & -8 \\
\hline Cyprus & 3,511 & 29 & 28 & 1 & $-8,609$ & 23 & 57 & -34 \\
\hline Albania & 11,596 & 63 & 52 & 11 & $8,041^{* *}$ & 65 & $57^{* * * *}$ & $8 * * * * * *$ \\
\hline Bolivia & 11,639 & 74 & 70 & 4 & 9,113 & 67 & 65 & 2 \\
\hline Kuwait & 12,669 & 40 & 27 & 13 & $6,867^{* *}$ & 32 & $28^{* * * * *}$ & $4^{* * * * * * *}$ \\
\hline Nicaragua & 10,079 & 77 & 75 & 2 & $6,722^{*}$ & $77^{* * *}$ & 70 & $7 * * * * *$ \\
\hline Macedonia & 5,829 & 54 & 53 & 1 & 1,571 & $51^{* * *}$ & 68 & $-17 * * * * *$ \\
\hline Singapore & 6,667 & 26 & 22 & 4 & $4,721^{*}$ & $27^{* * *}$ & 17 & $10^{* * * * *}$ \\
\hline Tajikistan & 3,148 & 73 & 76 & -3 & 1,309 & 70 & 73 & -3 \\
\hline Uzbekistan & $-2,338$ & 65 & 72 & -7 & 0,165 & 63 & 71 & -8 \\
\hline Peru & 7,898 & 60 & 57 & 3 & $5,375^{*}$ & $58 * * *$ & 52 & $6 * * * * *$ \\
\hline Colombia & 4,534 & 53 & 56 & -3 & 5,280 & 54 & 56 & -2 \\
\hline Philippines & $-0,398$ & 56 & 66 & -10 & 1,048 & 53 & 61 & -8 \\
\hline Indonesia & 7,310 & 72 & 68 & 4 & 4,048 & 66 & 67 & -1 \\
\hline Egypt & 9,502 & 75 & 74 & 1 & 6,749 & 69 & 72 & -3 \\
\hline El Salvador & 5,807 & 69 & 67 & 2 & 3,889 & 60 & 62 & -2 \\
\hline Honduras & 14,734 & 76 & 69 & 7 & $6,957^{*}$ & $75^{* * *}$ & 64 & $11^{* * * * * *}$ \\
\hline $\begin{array}{c}\text { Dominican } \\
\text { Republic }\end{array}$ & 6,878 & 65 & 63 & 2 & 4,406 & 62 & 63 & -1 \\
\hline Bahrain & 7,702 & 36 & 32 & 4 & 5,205 & 30 & 30 & 0 \\
\hline Kazakhstan & 7,200 & 57 & 60 & -3 & $4,505^{*}$ & $57^{* * *}$ & 53 & $4^{* * * * * *}$ \\
\hline Brazil & 7,398 & 55 & 55 & 0 & $3,739^{*}$ & $54^{* * *}$ & 51 & $3 * * * * *$ \\
\hline
\end{tabular}

*Growth between 2001 and 2007

**Growth between 2000 and 2006

***E-HDI Ranking in 2001

****E-HDI Ranking in 2006

*****Change in E-HDI Ranking between 2001 and 2007

****** Change in E-HDI Ranking between 2000 and 2006

Table 3: Employment-adjusted Human Development Index (E-HDI) Trends: 2000-2007 
developed (EAD), 3 are highly EAD and 4 are moderately EAD. The two African countries are moderately EAD (See Table 2).

During the period under study, 10 countries (i.e. Turkey, Ukraine, Bulgaria, Georgia Colombia, Albania, Paraguay, China and Ecuador) were promoted to being highly EAD from being moderately EAD. Likewise, 8 countries (i.e. Malta, Kuwait, Bahrain, Spain, Greece, Korean Republic, Czech Republic and Estonia) were elevated to being very highly EAD from highly EAD. Only Cyprus declined in its status from being very highly to highly EAD (See Tables 1 and 2).

The analysis shows that the E-HDI and HDI have classified some countries in different categories in terms of human development. In 2007, while Bahrain and Estonia are highly developed countries according to the $\mathrm{HDI}$, they are found to be very highly developed according to the E-HDI. While Ukraine, China, Paraguay and Georgia are moderately developed countries according to the $\mathrm{HDI}$, they are found to be highly developed according to the E-HDI. Similarly, while Tajikistan, Nicaragua, South Africa are low developed countries according to the HDI where the absolute values are lower than 0.700 , they are found to be moderately developed according to the E-HDI.

Likewise, while Macedonia is highly developed according to the $\mathrm{HDI}$, it is found to be moderately developed according to the E-HDI. Similarly, Cyprus is very highly developed according to the $\mathrm{HDI}$, and is found to be only highly developed according to the E-HDI.

Furthermore, there has been very small fluctuation in the E-HDIs of Uzbekistan, Kyrgyzstan, United States and South Africa with an E-HDI growth rate of less than $1 \%$. High E-HDI growth rates of more than $7.5 \%$ were experienced by Lithuania, Latvia, Estonia, Croatia, Ecuador, Bolivia, Chile and Albania. Only Cyprus and South Africa have experienced negative E-HDI growth rates during the period under investigation (See Table 3).

According to the present calculations, while Hungary, Slovakia, Uzbekistan and Phillipines experienced negative $\mathrm{HDI}$ growth rates during the analysis period, the E-HDI growth rates of these countries are found to be positive. Lastly, out of 77 countries, the E-HDI of only 22 countries was found to be greater than their HDIs.

\subsection{Analysis of OECD Countries}

The sample included 30 OECD countries, most of which are highly developed and developed countries.
Norway has led OECD countries for six years. According to both the $\mathrm{HDI}$ and $\mathrm{E}-\mathrm{HDI}$, it has always ranked within the top two countries.

Nineteen OECD countries consistenly had an E-HDI score greater than 0.900 , whereas only five countries (i.e. Hungary, Poland, Slovakia, Mexico and Turkey) among OECD countries consistently had an E-HDI score of less than 0.900 . The relatively less developed nine countries (Greece, the Republic of Korea, Portugal, Czech Republic, Hungary, Poland, Slovakia, Mexico and Turkey) constitute a "low category" with respect to the E-HDI, by ranking always below the other 20 OECD countries. Five countries (i.e. Hungary, Poland, Slovakia, Mexico and Turkey) always ranked in the last five among OECD countries.

Being the least developed OECD country, the E-HDI of Turkey has become greater than 0.800 only after 2005/6. This positive trend can be related to the significant rise in its employment rate alongside per capita growth in GDP and improvement in educational attainment figures (See Table 2 and 8).

The other cluster of OECD countries which could be categorized as "middle" ranked always between the 13rd and 22th among the 30 OECD countries. These six countries are New Zealand, Italy, Germany, France, Finland and Spain.

The "High" E-HDI category included Iceland, Norway, Australia, Canada, Ireland, Sweden, Switzerland and Japan since these countries always ranked in the top 11 OECD countries during the analysis period.

Switzerland and Austria have had mostly stable rankings during the analysis period, whereas the most unstable rankings were observed for Ireland and Denmark. Furthermore, Ireland and the Unites States had a similar range of rankings. Likewise, Finland and New Zealand has had the same range of rankings between the 13th and 18th. The United Kingdom experienced a drastic fall in its 2007 rankings. Likewise, Luxembourg had a drastic fall in its 2005/6 rankings.

Between 2005/6 and 2007, the E-HDI decreased only for Spain and the UK (See Table 2 and 8). Between 2004 and 2005, the E-HDI only decreased for Portugal (See Table 7 and 8). This trend can be associated with the signs of the global economic crisis for these countries. As is well known, Spain, Portugal and UK were adversely affected from the recent economic crisis, which manifested itself through declines in GDP, and especially a drastic rise in unemployment rates ${ }^{2}$.

\footnotetext{
2 These three countries are among those which heavily suffered from the recent financial crises. According to Reinhart and Rogoff (2009),
} 
The highest E-HDI value (0.972) was achieved by Norway in 2007. Spain, the Republic of Korea and Portugal reached 0.900 in 2000, Italy in 1999, Greece in 2002, and the Czech Republic in 2005/6.

Among OECD countries, highest loss of rankings (-8) was experienced by Denmark during 2000-2001, whereas the highest gain in ranking (+7) was achieved by Belgium during the same period (See Table 1 and 4).

The Highest annual increase in E-HDI was achieved by Turkey with 3.0\% between 2005/6-2007 (See Table 2 and 8). Similarly, the highest annual decrease in the E-HDI was again achieved by Turkey, with $-1.6 \%$ during the 2000 2001 period (See Table 1 and 4) owing to the most destructive crisis that the country ever experienced in its economic history. Consequently, severe unemployment problems deteriorated the human development performance of the country.

None of the OECD countries enjoyed continuous increases in its ranking. However, 13 OECD countries (i.e. Ireland, Japan, Finland, Spain, Denmark, Austria, United Kingdom, New Zealand, Italy, Greece, the Republic of Korea, Hungary and Poland) had a rising E-HDI trend during the analysis period. Ireland succeeded in increasing its ranking from 2000 through 2005/6. Nevertheless, the global economic crisis jeopardized this trend and negatively affected its performance in terms of human development (See Table 1, 2, 4, 5, 6, 7 and 8) . $^{3}$.

The E-HDI is greater than the $\mathrm{HDI}$ during the whole period for eight OECD countries, namely the Republic of Korea, New Zealand, Portugal, Turkey, Iceland, Japan, Mexico, Switzerland and Denmark. These countries had been underestimated by the HDI in terms of their human development levels. The addition of the employment factor to the HDI had significant effects on the values of the relatively less developed countries, such as Turkey, Mexico, the Republic of Korea and Portugal. Furthermore, Canada, France, Finland, Spain, Belgium and Italy had higher HDIs than E-HDls for all years except 2005/6, which could be considered the starting period of the recent global economic crisis.

\subsection{Analysis of Developing Countries}

In addition to 30 OECD countries, the sample also consisted of 44 developing and 3 highly developed (i.e.

severe financial crises have adverse and lasting effects on output and unemployment.

${ }^{3}$ To examine the effects of the current economic crisis on the basic fundamentals of the Irish economy, see for example Lane (2011), Hardiman (2010) and Kelly (2010).
Singapore, Israel and Hong Kong) countries. According to the E-HDI and HDI statistics, Kuwait began classed as very highly developed (i.e. HDI and E-HDI values exceeded 0.900 ) in 2004 and 2007 respectively, mainly due to its high per capita income level originating from the export revenues of oil products ${ }^{4}$. Additionally, the E-HDI and $\mathrm{HDI}$ statistics also depicted Malta as a very highly developed country with respect to human development in 2007 (See Table 2 and 7).

The countries with the highest HDI growth rate between 2000 and 2007 were Honduras (14.734\%), Kuwait (12.669\%), Bolivia (11.639\%), Albania (11.596\%), Ecuador (10.109\%) and Nicaragua (10.079\%). The country with the lowest HDI growth rate was found to be Uzbekistan (-2.338\%).

In 2000, there was no developing country in the sample which had an $\mathrm{HDI}$ higher than its E-HDI. Argentina's HDI exceeded its E-HDI during the 2001-2004 period. For all years under study, Israel and Macedonia had HDIs higher than E-HDIs. In 2001, Lithuania's E-HDI was equal to its HDI.

Bahrain, Romania, Indonesia, Trinidad and Ukraine have had mostly stable rankings during the analysis period. Controversially, developing countries like Macedonia, Cyprus and Cuba have had the most fluctuating rankings in their E-HDI.

The countries with the highest E-HDI growth rates during the analysis period were found to be Lithuania $(9.582 \%)$, Bolivia (9.113\%), Latvia (8.722\%), Bulgaria (8.624\%) and Albania (8.041\%). The country with the lowest E-HDI growth rate was found to be Cyprus. In fact, Cyprus had a negative growth rate during the analysis period (-8.609\%).

Furthermore, the biggest gains in E-HDI rankings between 2000 and 2007 were realised by Lithuania $(+12)$, Honduras $(+11)$, Singapore $(+10)$, Ecuador (+9), Latvia $(+8)$, Cuba (+8), Albania (+8), Estonia (+7) and Nicaragua $(+7)$. The biggest drops in E-HDI rankings in this period were experienced by Cyprus (-34), Macedonia (-17), Phillipines (-8) and Kyrgyzstan (-8) (See Table 3).

Depending on the comparison between $\mathrm{HDI}$ and $\mathrm{E}-$ $\mathrm{HDI}$ rankings, it can be argued that at the beginning of the analysis period (in 2000), the current HDI underestimated the rankings of China $(+12)$, Belarus $(+10)$

\footnotetext{
${ }^{4}$ According to Wikipedia, Kuwait is a small economy with crude oil reserves of about 96 billion barrels which constitute nearly nine per cent of the total world oil reserves. In turn, oil accounts for about half of GDP, and 90 per cent of export revenues (http://en.wikipedia.org/wiki/Economy_of_Kuwait).
} 
and El Salvador (+9), whereas Slovakia, Lithuania and Croatia were overestimated by $+6,+4$ and +4 rankings, respectively (Table 1 ). For the following year, the highest negative difference between the rankings of $\mathrm{HDI}$ and EHDI were observed for Belarus (-11), Kuwait (-10), China (-9), Malaysia (-9) and Cuba (-9). Macedonia gained 15 places (Table 4).

In 2002, however, the highest negative differences between the rankings of the $\mathrm{HDI}$ and $\mathrm{E}-\mathrm{HDI}$ were observed for Macedonia (-17), Argentina (-8) and Bulgaria $(-7)$. Cuba, Kuwait and Malaysia gained $+9,+8$ and +8 places, respectively (Table 5). In 2005-6, the highest difference between the rankings of the $\mathrm{HDI}$ and $\mathrm{E}-\mathrm{HDI}$ was observed for Macedonia (-18). Cuba gained 6 places. The rankings of Cyprus, Moldova, Malta, Russia and Georgia remained the same (Table 7).

At the terminal year of the analysis period (in 2007), addition of the unemployment factor to the HDI has caused Cyprus and Macedonia to lose (-29) and (-15) places, respectively. On the other hand, Cuba and Belarus have gained +12 and +8 places, respectively (Table 8 ). This brief descriptive analysis indicates that addition of the unemployment factor to the HDI affected Macedonia the most among developing countries. This effect remained mostly stable after 2001.

\section{Conclusion}

This paper on the Human Development Index represents a first attempt to construct a measure of human development for 77 countries based on the framework developed incorporating the human security component. A new index on human development, the E$\mathrm{HDI}$, is defined as the average of the scaled value of the four components: income, human security, health and education. While sub-indices of income, health and education are taken from the UNDP's Human Development Reports, the total unemployment rate has been pointed to and used as an indicator of human security, i.e. the new proposed sub-index. In doing so, the current study searches for whether the inclusion of an employment indicator into the existing HDI would result in different rankings of countries. Additionally, it is disputed that the HDI could be appropriately altered via integrating an employment dimension to the current index.

The experiences of a relatively large number of 77 sample countries offer promising results for the path to improve the current status of the $\mathrm{HDI}$, and hence, to overcome some of its weaknesses. Above all, the dimension of the current index could be enriched with the E-HDI. An additional indicator obviously improves the explanatory power of the HDI and makes significant contributions to its reliability. Moreover, the human development performance of countries could be better evaluated and compared with other countries by using additional information obtained from the employment situations of their citizens.

However, it should be mentioned that the present study could further be ameliorated through assigning each component a weighting depending on any predetermined criteria rather than assigning these on an arbitrary basis. Future research may focus on this field.

A major limitation of the present study has been the lack of data for a number of the human development indicators for the rest of the countries in the world. Future work will hopefully address these gaps and permit the development of more comprehensive and reliable measurement of the various components of the HDI as better data sources are identified and data accessed. Nevertheless, we believe that the current research, despite its exploratory nature, provides significant insight on evaluating and ranking countries over the last decade.

ㅁ.

\section{References}

Akyüz, Y. and Boratav, K. (2003), "The Making of the Turkish Financial Crisis", World Development, 31 (9): 1549-1566.

Cherchye, L., Ooghe, E. and Van Puyenbroeck, T. (2008), "Robust Human Development Rankings", Journal of Economic Inequality, 6 (4): 287-321.

Dufour, M. and Orhangazi, Ö (2009), "The 2000-2001 Financial Crisis in Turkey: A Crisis for Whom?", Review of Political Economy, 21 (1): 101-122.

Ghosh, P. K. (1984), Third World Development: A Basic Need Approach, Westport, Conn.: Greenwood Press.

Hardiman, N. (2010), "Bringing Domestic Institutions back into an Understanding of Ireland's Economic Crisis", Irish Studies in International Affairs, 21, 71-87.

Hicks, N. and Streeten, P. (1979), "Indicators of Development: The Search of a Basic needs Yardstick", World Development, 7: 567-580.

ILO (International Labour Organization), LABORSTA - database of labour statistics, http://laborsta.ilo.org/

ILO (International Labour Organization) (1976), Employment, Growth, and Basic Needs, Geneva: ILO.

Kelly, M. (2010), "Whatever Happened to Ireland?" CEPR Discussion Paper No. DP7811. Available at SSRN: http://ssrn.com/abstract=1611507

Lane, P. R. (2011), "The Irish Crisis", CEPR Discussion Paper No. DP8287. Available at SSRN: http://ssrn.com/abstract=1794877.

Osberg, L. and Sharpe, A. (2003), "Human Well-being and Economic Well-being: What Values Are Implicit in Current Indices?", Centre for the Study of Living Standards Research Report 2003/04, Ottowa: Centre for the Study of Living Standards. 
Öniş, Z. (2009), "Beyond the 2001 Financial Crisis: The Political Economy of the New Phase of Neo-Liberal Restructuring in Turkey", Review of International Political Economy, 16 (3): 409-432.

Panigrahi, R. and Sivramkrishna, S. (2002), "An adjusted Human Development Index: Robust Country Rankings with Respect to the Choice of Fixed Maximum and Minimum Indicator Values", Journal of Human Development, 3 (2): 301-311.

Reinhart, M.C. and Rogoff, K.S. (2009), "The Aftermath of Financial Crises", American Economic Review, 99 (2): 466-472.

Sen, A. (1983), "Development: Which Way Now?", Economic Journal, 93: 745-762.

Stewart, F. (1985), Planning to Meet Basic Needs, London: Macmillan Publications.

Stewart, F. (1995), "Basic Needs, Capabilities and Human Development", Greek Economic Review, 17 (2): 83-96.

Şenses, F. (2003), "Economic Crisis as an Instigator of Distributional Conflict: The Turkish Case in 2001", Turkish Studies, 4 (2): 92-119.

Taner, M. T., Sezen, B., Alpkan, L. and Aren, S. (2010), Recomputation of UNDP's HDI rankings by Data Envelopment Analysis. VI. International Strategic Management Conference, St.Petersburg, Russia, July.

Taner, M. T., Sezen, B. and Mıhcl, H. (2011), "An Alternative Human Development Index Considering Unemployment", South East European Journal of Economics and Business, 6 (1): 45- 60.

UNDP (1994), Human Development Report 1994, New York: Oxford University Press.

UNDP (2002), Human Development Report 2002, New York: Oxford University Press.

UNDP (2003), Human Development Report 2003, New York: Oxford University Press.

UNDP (2004), Human Development Report 2004, New York: Oxford University Press.

UNDP (2005), Human Development Report 2005, New York: Oxford University Press.

UNDP (2006), Human Development Report 2006, New York: Oxford University Press.

UNDP (2007-2008), Human Development Report 2007-2008, New York: Oxford University Press.

UNDP (2009), Human Development Report 2009, New York: Oxford University Press.

Wolff, H., Chong H. and Auffhammer, M. (2009), Human Development Index: Are Developing Countries Misclassified?. Agricultural and Applied Economics Association Annual Meeting, Milwaukee, Wisconsin.

Yeldan, E. (2006), "Neoliberal Global Remedies: From Speculative-Led Growth to IMF-Led Crisis in Turkey", Review of Radical Political Economics, 38 (2): 193-213. 
Appendix

\begin{tabular}{|c|c|c|c|c|c|c|c|c|c|c|}
\hline & LEI & GDPI & EMPI & EI & E-HDI & HDI & HDI-E-HDI & R(HDI) & RANK-E-HDI & R(HDI-E-HDI) \\
\hline Iceland & 0,91 & 0,95 & 0,977 & 0,96 & 0,94925 & 0,942 & $-0,00725$ & 2 & 3 & -1 \\
\hline Norway & 0,90 & 0,95 & 0,965 & 0,99 & 0,95125 & 0,944 & $-0,00725$ & 1 & 1 & 0 \\
\hline Australia & 0,90 & 0,92 & 0,933 & 0,99 & 0,93575 & 0,939 & 0,00325 & 4 & 10 & -6 \\
\hline Canada & 0,90 & 0,94 & 0,928 & 0,97 & 0,9345 & 0,937 & 0,0025 & 6 & 14 & -8 \\
\hline Ireland & 0,86 & 0,96 & 0,961 & 0,96 & 0,93525 & 0,93 & $-0,00525$ & 11 & 11 & 0 \\
\hline Sweden & 0,91 & 0,92 & 0,96 & 0,99 & 0,945 & 0,941 & $-0,004$ & 3 & 4 & -1 \\
\hline Switzerland & 0,90 & 0,94 & 0,981 & 0,95 & 0,94275 & 0,932 & $-0,01075$ & 9 & 5 & 4 \\
\hline Japan & 0,94 & 0,92 & 0,95 & 0,94 & 0,9375 & 0,932 & $-0,0055$ & 9 & 9 & 0 \\
\hline Netherlands & 0,89 & 0,94 & 0,98 & 0,99 & 0,95 & 0,938 & $-0,012$ & 5 & 2 & 3 \\
\hline France & 0,90 & 0,91 & 0,913 & 0,96 & 0,92075 & 0,925 & 0,00425 & 17 & 19 & -2 \\
\hline Finland & 0,88 & 0,92 & 0,908 & 0,99 & 0,9245 & 0,93 & 0,0055 & 11 & 16 & -5 \\
\hline United States & 0,86 & 0,97 & 0,952 & 0,97 & 0,938 & 0,937 & $-0,001$ & 6 & 8 & -2 \\
\hline Spain & 0,90 & 0,89 & 0,895 & 0,97 & 0,91375 & 0,918 & 0,00425 & 19 & 20 & -1 \\
\hline Denmark & 0,86 & 0,95 & 0,957 & 0,99 & 0,93925 & 0,93 & $-0,00925$ & 11 & 6 & 5 \\
\hline Austria & 0,89 & 0,93 & 0,951 & 0,97 & 0,93525 & 0,929 & $-0,00625$ & 16 & 11 & 5 \\
\hline United Kingdom & 0,88 & 0,92 & 0,949 & 0,99 & 0,93475 & 0,93 & $-0,00475$ & 11 & 13 & -2 \\
\hline Belgium & 0,89 & 0,92 & 0,934 & 0,99 & 0,9335 & 0,937 & 0,0035 & 6 & 15 & -9 \\
\hline Luxembourg & 0,88 & 1 & 0,974 & 0,9 & 0,9385 & 0,93 & $-0,0085$ & 11 & 7 & 4 \\
\hline New Zealand & 0,88 & 0,88 & 0,947 & 0,99 & 0,92425 & 0,917 & $-0,00725$ & 20 & 17 & 3 \\
\hline Italy & 0,89 & 0,92 & 0,904 & 0,93 & 0,911 & 0,916 & 0,005 & 21 & 22 & -1 \\
\hline Germany & 0,88 & 0,92 & 0,927 & 0,96 & 0,92175 & 0,921 & $-0,00075$ & 18 & 18 & 0 \\
\hline Greece & 0,89 & 0,86 & 0,896 & 0,93 & 0,894 & 0,892 & $-0,002$ & 28 & 24 & 4 \\
\hline Republic of Korea & 0,84 & 0,84 & 0,963 & 0,96 & 0,90075 & 0,879 & $-0,02175$ & 27 & 28 & -1 \\
\hline Portugal & 0,85 & 0,87 & 0,959 & 0,97 & 0,91225 & 0,896 & $-0,01625$ & 23 & 21 & 2 \\
\hline Czech Republic & 0,83 & 0,83 & 0,918 & 0,91 & 0,872 & 0,861 & $-0,011$ & 30 & 29 & 1 \\
\hline Hungary & 0,77 & 0,8 & 0,942 & 0,93 & 0,8605 & 0,837 & $-0,0235$ & 32 & 34 & -2 \\
\hline Poland & 0,81 & 0,76 & 0,818 & 0,95 & 0,8345 & 0,841 & 0,0065 & 41 & 32 & 9 \\
\hline Slovakia & 0,80 & 0,8 & 0,807 & 0,9 & 0,82675 & 0,836 & 0,00925 & 44 & 35 & 9 \\
\hline Mexico & 0,80 & 0,74 & 0,975 & 0,86 & 0,84375 & 0,8 & $-0,04375$ & 36 & 47 & -11 \\
\hline Turkey & 0,75 & 0,68 & 0,915 & 0,77 & 0,77875 & 0,734 & $-0,04475$ & 61 & 64 & -3 \\
\hline Estonia & 0,77 & 0,77 & 0,874 & 0,96 & 0,8435 & 0,833 & $-0,0105$ & 37 & 38 & -1 \\
\hline Georgia & 0,81 & 0,54 & 0,89 & 0,89 & 0,7825 & 0,746 & $-0,0365$ & 60 & 61 & -1 \\
\hline Hong Kong & 0,91 & 0,92 & 0,949 & 0,83 & 0,90225 & 0,889 & $-0,01325$ & 26 & 26 & 0 \\
\hline Israel & 0,9 & 0,88 & 0,906 & 0,93 & 0,904 & 0,905 & 0,001 & 22 & 22 & 0 \\
\hline Latvia & 0,76 & 0,73 & 0,869 & 0,95 & 0,82725 & 0,811 & $-0,01625$ & 42 & 43 & -1 \\
\hline Lithuania & 0,79 & 0,74 & 0,826 & 0,94 & 0,824 & 0,824 & 0 & 46 & 40 & 6 \\
\hline Malaysia & 0,8 & 0,75 & 0,965 & 0,83 & 0,83625 & 0,79 & $-0,04625$ & 40 & 49 & -9 \\
\hline Argentina & 0,81 & 0,79 & 0,826 & 0,94 & 0,8415 & 0,849 & 0,0075 & 38 & 31 & 7 \\
\hline Bulgaria & 0,76 & 0,71 & 0,806 & 0,91 & 0,7965 & 0,795 & $-0,0015$ & 55 & 48 & 7 \\
\hline Chile & 0,85 & 0,75 & 0,921 & 0,89 & 0,85275 & 0,831 & $-0,02175$ & 33 & 39 & -6 \\
\hline Croatia & 0,82 & 0,75 & 0,842 & 0,88 & 0,823 & 0,818 & $-0,005$ & 47 & 42 & 5 \\
\hline China & 0,76 & 0,62 & 0,964 & 0,79 & 0,7835 & 0,721 & $-0,0625$ & 59 & 68 & -9 \\
\hline Russia & 0,69 & 0,71 & 0,911 & 0,93 & 0,81025 & 0,779 & $-0,03125$ & 49 & 52 & -3 \\
\hline Belarus & 0,74 & 0,72 & 0,977 & 0,95 & 0,84675 & 0,804 & $-0,04275$ & 34 & 45 & -11 \\
\hline Cuba & 0,86 & 0,66 & 0,959 & 0,9 & 0,84475 & 0,806 & $-0,03875$ & 35 & 44 & -9 \\
\hline Ecuador & 0,76 & 0,58 & 0,89 & 0,85 & 0,77 & 0,731 & $-0,039$ & 64 & 65 & -1 \\
\hline
\end{tabular}




\begin{tabular}{|c|c|c|c|c|c|c|c|c|c|c|}
\hline & LEI & GDPI & EMPI & EI & E-HDI & HDI & HDI-E-HDI & $R(H D I)$ & RANK-E-HDI & $R(H D I-E-H D I)$ \\
\hline Panama & 0,82 & 0,68 & 0,853 & 0,86 & 0,80325 & 0,788 & $-0,01525$ & 51 & 50 & 1 \\
\hline Romania & 0,76 & 0,68 & 0,934 & 0,88 & 0,8135 & 0,773 & $-0,0405$ & 48 & 55 & -7 \\
\hline Trinidad & 0,78 & 0,75 & 0,892 & 0,88 & 0,8255 & 0,802 & $-0,0235$ & 45 & 46 & -1 \\
\hline Ukraine & 0,74 & 0,63 & 0,891 & 0,93 & 0,79775 & 0,766 & $-0,03175$ & 54 & 56 & -2 \\
\hline Slovakia & 0,8 & 0,8 & 0,808 & 0,9 & 0,827 & 0,836 & 0,009 & 43 & 35 & 8 \\
\hline Paraguay & 0,76 & 0,66 & 0,924 & 0,84 & 0,796 & 0,751 & $-0,045$ & 56 & 59 & -3 \\
\hline Uruguay & 0,83 & 0,74 & 0,847 & 0,93 & 0,83675 & 0,834 & $-0,00275$ & 39 & 37 & 2 \\
\hline South Africa & 0,43 & 0,79 & 0,706 & 0,83 & 0,689 & 0,684 & $-0,005$ & 76 & 71 & 5 \\
\hline Malta & 0,88 & 0,81 & 0,936 & 0,87 & 0,874 & 0,856 & $-0,018$ & 29 & 30 & -1 \\
\hline Moldova & 0,72 & 0,51 & 0,932 & 0,86 & 0,7555 & 0,7 & $-0,0555$ & 69 & 70 & -1 \\
\hline Kyrgyzstan & 0,72 & 0,55 & 0,922 & 0,91 & 0,7755 & 0,727 & $-0,0485$ & 62 & 67 & -5 \\
\hline Cyprus & 0,88 & 0,89 & 0,96 & 0,9 & 0,9075 & 0,891 & $-0,0165$ & 23 & 25 & -2 \\
\hline Albania & 0,81 & 0,6 & 0,836 & 0,8 & 0,7615 & 0,735 & $-0,0265$ & 67 & 63 & 4 \\
\hline Bolivia & 0,64 & 0,52 & 0,915 & 0,85 & 0,73125 & 0,672 & $-0,05925$ & 72 & 74 & -2 \\
\hline Kuwait & 0,86 & 0,87 & 0,992 & 0,73 & 0,863 & 0,82 & $-0,043$ & 31 & 41 & -10 \\
\hline Nicaragua & 0,73 & 0,53 & 0,936 & 0,66 & 0,714 & 0,643 & $-0,071$ & 74 & 77 & -3 \\
\hline Macedonia & 0,81 & 0,69 & 0,695 & 0,86 & 0,76375 & 0,784 & 0,02025 & 66 & 51 & 15 \\
\hline Singapore & 0,88 & 0,91 & 0,962 & 0,87 & 0,9055 & 0,884 & $-0,0215$ & 24 & 27 & -3 \\
\hline Tajikistan & 0,72 & 0,41 & 0,832 & 0,9 & 0,7155 & 0,677 & $-0,0385$ & 73 & 73 & 0 \\
\hline Uzbekistan & 0,74 & 0,53 & 0,847 & 0,91 & 0,75675 & 0,729 & $-0,02775$ & 68 & 66 & 2 \\
\hline Peru & 0,74 & 0,64 & 0,921 & 0,88 & 0,79525 & 0,752 & $-0,04325$ & 57 & 58 & -1 \\
\hline Colombia & 0,78 & 0,71 & 0,854 & 0,85 & 0,7985 & 0,779 & $-0,0195$ & 53 & 52 & 1 \\
\hline Philippines & 0,74 & 0,61 & 0,889 & 0,9 & 0,78475 & 0,751 & $-0,03375$ & 58 & 59 & -1 \\
\hline Indonesia & 0,69 & 0,56 & 0,919 & 0,8 & 0,74225 & 0,682 & $-0,06025$ & 70 & 72 & -2 \\
\hline Egypt & 0,72 & 0,59 & 0,908 & 0,63 & 0,712 & 0,648 & $-0,064$ & 75 & 76 & -1 \\
\hline El Salvador & 0,76 & 0,66 & 0,93 & 0,74 & 0,7725 & 0,719 & $-0,0535$ & 63 & 69 & -6 \\
\hline Honduras & 0,73 & 0,56 & 0,961 & 0,71 & 0,74025 & 0,667 & $-0,07325$ & 71 & 75 & -4 \\
\hline Dominican Republic & 0,7 & 0,71 & 0,844 & 0,81 & 0,766 & 0,737 & $-0,029$ & 65 & 62 & 3 \\
\hline Bahrain & 0,81 & 0,85 & $n / a$ & 0,86 & $\mathrm{n} / \mathrm{a}$ & 0,839 & $\mathrm{n} / \mathrm{a}$ & $n / a$ & 33 & $n / a$ \\
\hline Kazakhstan & 0,68 & 0,7 & 0,896 & 0,92 & 0,799 & 0,765 & $-0,034$ & 52 & 57 & -5 \\
\hline Brazil & 0,71 & 0,72 & 0,906 & 0,9 & 0,809 & 0,777 & $-0,032$ & 50 & 54 & -4 \\
\hline
\end{tabular}

EMPI, E-HDI, R(E-HDI): Authors' own calculations

Source: UNDP (2003); ILO

Table 4: Employment-adjusted Human Development Index (E-HDI): 2001

\begin{tabular}{|c|c|c|c|c|c|c|c|c|c|c|}
\hline & LEI & GDPI & EMPI & EI & E-HDI & HDI & HDI-E-HDI & R(HDI) & R(E-HDI) & R(HDI-E-HDI) \\
\hline Iceland & 0,91 & 0,95 & 0,967 & 0,96 & 0,94675 & 0,941 & $-0,00575$ & 7 & 4 & 3 \\
\hline Norway & 0,90 & 0,99 & 0,96 & 0,99 & 0,96 & 0,956 & $-0,004$ & 1 & 1 & 0 \\
\hline Australia & 0,90 & 0,94 & 0,937 & 0,99 & 0,94175 & 0,946 & 0,00425 & 3 & 7 & -4 \\
\hline Canada & 0,90 & 0,95 & 0,924 & 0,98 & 0,9385 & 0,943 & 0,0045 & 4 & 13 & -9 \\
\hline Ireland & 0,86 & 0,98 & 0,956 & 0,96 & 0,939 & 0,936 & $-0,003$ & 10 & 10 & 0 \\
\hline Sweden & 0,92 & 0,93 & 0,96 & 0,99 & 0,95 & 0,946 & $-0,004$ & 2 & 3 & -1 \\
\hline Switzerland & 0,90 & 0,95 & 0,969 & 0,95 & 0,94225 & 0,936 & $-0,00625$ & 10 & 6 & 4 \\
\hline Japan & 0,94 & 0,93 & 0,946 & 0,94 & 0,939 & 0,938 & $-0,001$ & 9 & 11 & -2 \\
\hline Netherlands & 0,89 & 0,95 & 0,977 & 0,99 & 0,95175 & 0,942 & $-0,00975$ & 5 & 2 & 3 \\
\hline France & 0,90 & 0,93 & 0,91 & 0,96 & 0,925 & 0,932 & 0,007 & 18 & 18 & 0 \\
\hline Finland & 0,88 & 0,93 & 0,909 & 0,99 & 0,92725 & 0,935 & 0,00775 & 13 & 17 & -4 \\
\hline
\end{tabular}


Employment-adjusted Human Development Index

\begin{tabular}{|c|c|c|c|c|c|c|c|c|c|c|}
\hline & LEI & GDPI & EMPI & EI & E-HDI & HDI & HDI-E-HDI & $\mathbf{R}(\mathrm{HDI})$ & $R$ (E-HDI) & R(HDI-E-HDI) \\
\hline United States & 0,87 & 0,98 & 0,942 & 0,97 & 0,9405 & 0,939 & $-0,0015$ & 8 & 8 & 0 \\
\hline Spain & 0,89 & 0,9 & 0,886 & 0,97 & 0,9115 & 0,922 & 0,0105 & 20 & 22 & -2 \\
\hline Denmark & 0,86 & 0,96 & 0,955 & 0,98 & 0,93875 & 0,932 & $-0,00675$ & 16 & 12 & 4 \\
\hline Austria & 0,89 & 0,95 & 0,947 & 0,96 & 0,93675 & 0,934 & $-0,00275$ & 14 & 15 & -1 \\
\hline United Kingdom & 0,88 & 0,93 & 0,948 & 0,99 & 0,937 & 0,936 & $-0,001$ & 10 & 14 & -4 \\
\hline Belgium & 0,90 & \begin{tabular}{|l|}
0,94 \\
\end{tabular} & 0,927 & 0,99 & 0,93925 & 0,942 & 0,00275 & 5 & 9 & -4 \\
\hline Luxembourg & 0,89 & 1 & 0,97 & 0,91 & 0,9425 & 0,933 & $-0,0095$ & 15 & 5 & 10 \\
\hline New Zealand & 0,89 & 0,9 & 0,948 & 0,99 & 0,932 & 0,926 & $-0,006$ & 18 & 16 & 2 \\
\hline Italy & 0,89 & 0,93 & 0,909 & 0,93 & 0,91475 & 0,92 & 0,00525 & 21 & 20 & 1 \\
\hline Germany & 0,89 & 0,94 & 0,919 & 0,95 & 0,92475 & 0,925 & 0,00025 & 19 & 19 & 0 \\
\hline Greece & 0,89 & 0,87 & 0,9 & 0,95 & 0,9025 & 0,902 & $-0,0005$ & 24 & 28 & -4 \\
\hline Republic of Korea & 0,84 & 0,86 & 0,969 & 0,97 & 0,90975 & 0,888 & $-0,02175$ & 27 & 23 & 4 \\
\hline Portugal & 0,85 & 0,87 & 0,949 & 0,97 & 0,90975 & 0,897 & $-0,01275$ & 26 & 23 & 3 \\
\hline Czech Republic & 0,84 & 0,84 & 0,927 & 0,92 & 0,88175 & 0,868 & $-0,01375$ & 30 & 30 & 0 \\
\hline Hungary & 0,78 & 0,82 & 0,941 & 0,95 & 0,87275 & 0,848 & $-0,02475$ & 34 & 32 & 2 \\
\hline Poland & 0,81 & 0,78 & 0,801 & 0,96 & 0,83775 & 0,85 & 0,01225 & 33 & 40 & -7 \\
\hline Slovakia & 0,81 & 0,81 & 0,814 & 0,91 & 0,836 & 0,842 & 0,006 & 36 & 43 & -7 \\
\hline Mexico & 0,81 & 0,75 & 0,973 & 0,85 & 0,84575 & 0,802 & $-0,04375$ & 45 & 37 & 8 \\
\hline Turkey & 0,76 & 0,69 & 0,897 & 0,8 & 0,78675 & 0,751 & $-0,03575$ & 61 & 60 & 1 \\
\hline Estonia & 0,78 & 0,8 & 0,897 & 0,98 & 0,86425 & 0,853 & $-0,01125$ & 31 & 33 & -2 \\
\hline Georgia & 0,81 & 0,52 & 0,877 & 0,89 & 0,77425 & 0,739 & $-0,03525$ & 64 & 65 & -1 \\
\hline Hong Kong & 0,91 & 0,93 & 0,927 & 0,86 & 0,90675 & 0,903 & $-0,00375$ & 23 & 25 & -2 \\
\hline Israel & 0,9 & 0,88 & 0,897 & 0,94 & 0,90425 & 0,908 & 0,00375 & 22 & 26 & -4 \\
\hline Latvia & 0,76 & 0,75 & 0,88 & 0,95 & 0,835 & 0,823 & $-0,012$ & 43 & 45 & -2 \\
\hline Lithuania & 0,79 & 0,77 & 0,862 & 0,96 & 0,8455 & 0,842 & $-0,0035$ & 36 & 38 & -2 \\
\hline Malaysia & 0,8 & 0,75 & 0,965 & 0,83 & 0,83625 & 0,793 & $-0,04325$ & 49 & 41 & 8 \\
\hline Argentina & 0,82 & 0,78 & 0,804 & 0,96 & 0,841 & 0,853 & 0,012 & 31 & 39 & -8 \\
\hline Bulgaria & 0,77 & \begin{tabular}{|l|}
0,71 \\
\end{tabular} & 0,824 & 0,91 & 0,8035 & 0,796 & $-0,0075$ & 47 & 54 & -7 \\
\hline Chile & 0,85 & 0,77 & 0,922 & 0,9 & 0,8605 & 0,839 & $-0,0215$ & 39 & 34 & 5 \\
\hline Croatia & 0,82 & \begin{tabular}{|l|}
0,77 \\
\end{tabular} & 0,852 & 0,9 & 0,8355 & 0,83 & $-0,0055$ & 42 & 44 & -2 \\
\hline China & 0,76 & 0,64 & 0,96 & 0,83 & 0,7975 & 0,745 & $-0,0525$ & 63 & 57 & 6 \\
\hline Russia & 0,69 & 0,74 & 0,921 & 0,95 & 0,82525 & 0,795 & $-0,03025$ & 48 & 48 & 0 \\
\hline Belarus & 0,75 & \begin{tabular}{|l|}
0,67 \\
\end{tabular} & 0,97 & 0,95 & 0,835 & 0,79 & $-0,045$ & 52 & 45 & 7 \\
\hline Cuba & 0,86 & 0,66 & 0,967 & 0,91 & 0,84925 & 0,809 & $-0,04025$ & 44 & 36 & 8 \\
\hline Ecuador & 0,76 & 0,6 & 0,907 & 0,85 & 0,77925 & 0,735 & $-0,04425$ & 66 & 63 & 3 \\
\hline Panama & 0,83 & 0,69 & 0,859 & 0,86 & 0,80975 & 0,791 & $-0,01875$ & 51 & 51 & 0 \\
\hline Romania & 0,76 & 0,7 & 0,916 & 0,88 & 0,814 & 0,778 & $-0,036$ & 54 & 50 & 4 \\
\hline Trinidad & 0,77 & 0,76 & 0,896 & 0,87 & 0,824 & 0,801 & $-0,023$ & 46 & 49 & -3 \\
\hline Ukraine & 0,74 & 0,65 & 0,904 & 0,94 & 0,8085 & 0,777 & $-0,0315$ & 55 & 53 & 2 \\
\hline Slovakia & 0,81 & 0,81 & 0,815 & 0,91 & 0,83625 & 0,842 & 0,00575 & 36 & 41 & -5 \\
\hline Paraguay & 0,76 & 0,64 & 0,892 & 0,85 & 0,7855 & 0,751 & $-0,0345$ & 61 & 62 & -1 \\
\hline Uruguay & 0,84 & 0,73 & 0,83 & 0,94 & 0,835 & 0,833 & $-0,002$ & 41 & 45 & -4 \\
\hline South Africa & 0,4 & 0,77 & 0,696 & 0,83 & 0,674 & 0,666 & $-0,008$ & 76 & 77 & -1 \\
\hline Malta & 0,89 & 0,86 & 0,93 & 0,87 & 0,8875 & 0,875 & $-0,0125$ & 29 & 29 & 0 \\
\hline Moldova & 0,73 & 0,45 & 0,921 & 0,87 & 0,74275 & 0,681 & $-0,06175$ & 71 & 71 & 0 \\
\hline Kyrgyzstan & 0,72 & 0,46 & 0,875 & 0,92 & 0,74375 & 0,701 & $-0,04275$ & 69 & 69 & 0 \\
\hline Cyprus & 0,89 & 0,87 & 0,967 & 0,89 & 0,90425 & 0,883 & $-0,02125$ & 28 & 26 & 2 \\
\hline Albania & 0,81 & 0,65 & 0,842 & 0,89 & 0,798 & 0,781 & $-0,017$ & 53 & 56 & -3 \\
\hline
\end{tabular}




\begin{tabular}{|c|c|c|c|c|c|c|c|c|c|c|}
\hline & LEI & GDPI & EMPI & EI & E-HDI & HDI & HDI-E-HDI & R(HDI) & R(E-HDI) & R(HDI-E-HDI) \\
\hline Bolivia & 0,64 & 0,53 & 0,913 & 0,86 & 0,73575 & 0,681 & $-0,05475$ & 71 & 73 & -2 \\
\hline Kuwait & 0,86 & 0,85 & 0,989 & 0,81 & 0,87725 & 0,838 & $-0,03925$ & 40 & 31 & 9 \\
\hline Nicaragua & 0,74 & 0,54 & 0,878 & 0,73 & 0,722 & 0,667 & $-0,055$ & 75 & 74 & 1 \\
\hline Macedonia & 0,81 & 0,7 & 0,681 & 0,87 & 0,76525 & 0,793 & 0,02775 & 49 & 66 & -17 \\
\hline Singapore & 0,88 & 0,92 & 0,944 & 0,91 & 0,9135 & 0,902 & $-0,0115$ & 24 & 21 & 3 \\
\hline Tajikistan & 0,73 & 0,38 & 0,803 & 0,9 & 0,70325 & 0,671 & $-0,03225$ & 74 & 76 & -2 \\
\hline Uzbekistan & 0,74 & 0,47 & 0,83 & 0,91 & 0,7375 & 0,709 & $-0,0285$ & 68 & 72 & -4 \\
\hline Peru & 0,74 & 0,65 & 0,923 & 0,86 & 0,79325 & 0,752 & $-0,04125$ & 60 & 58 & 2 \\
\hline Colombia & 0,78 & 0,69 & 0,848 & 0,84 & 0,7895 & 0,773 & $-0,0165$ & 57 & 59 & -2 \\
\hline Philippines & 0,75 & 0,62 & 0,886 & 0,89 & 0,7865 & 0,753 & $-0,0335$ & 59 & 61 & -2 \\
\hline Indonesia & 0,69 & 0,58 & 0,909 & 0,8 & 0,74475 & 0,692 & $-0,05275$ & 70 & 68 & 2 \\
\hline Egypt & 0,73 & 0,61 & 0,898 & 0,62 & 0,7145 & 0,653 & $-0,0615$ & 77 & 75 & 2 \\
\hline El Salvador & 0,76 & 0,65 & 0,938 & 0,75 & 0,7745 & 0,72 & $-0,0545$ & 67 & 64 & 3 \\
\hline Honduras & 0,73 & 0,54 & 0,962 & 0,74 & 0,743 & 0,672 & $-0,071$ & 73 & 70 & 3 \\
\hline Dominican Republic & 0,7 & 0,7 & 0,839 & 0,82 & 0,76475 & 0,738 & $-0,02675$ & 65 & 67 & -2 \\
\hline Bahrain & 0,81 & 0,86 & 0,913 & 0,85 & 0,85825 & 0,843 & $-0,01525$ & 35 & 35 & 0 \\
\hline Kazakhstan & 0,69 & 0,68 & 0,907 & 0,93 & 0,80175 & 0,766 & $-0,03575$ & 58 & 55 & 3 \\
\hline Brazil & 0,72 & 0,73 & 0,908 & 0,88 & 0,8095 & 0,775 & $-0,0345$ & 56 & 51 & 5 \\
\hline
\end{tabular}

EMPI, E-HDI, R(E-HDI): Authors' own calculations

Source: UNDP (2004); ILO

Table 5: Employment-adjusted Human Development Index (E-HDI): 2002

\begin{tabular}{|c|c|c|c|c|c|c|c|c|c|c|}
\hline & LEI & GDPI & EMPI & EI & E-HDI & HDI & HDI-E-HDI & R(E-HDI) & R(HDI) & R(HDI-E-HDI) \\
\hline Iceland & 0,93 & 0,96 & 0,955 & 0,98 & 0,95625 & 0,956 & $-0,00025$ & 2 & 2 & 0 \\
\hline Norway & 0,91 & 0,99 & 0,966 & 0,99 & 0,964 & 0,963 & $-0,001$ & 1 & 1 & 0 \\
\hline Australia & 0,92 & 0,95 & 0,94 & 0,99 & 0,95 & 0,955 & 0,005 & 5 & 3 & -2 \\
\hline Canada & 0,92 & 0,96 & 0,924 & 0,97 & 0,9435 & 0,949 & 0,0055 & 10 & 4 & -6 \\
\hline Ireland & 0,88 & 0,99 & 0,954 & 0,97 & 0,9485 & 0,946 & $-0,0025$ & 6 & 8 & 2 \\
\hline Sweden & 0,92 & 0,93 & 0,951 & 0,99 & 0,94775 & 0,949 & 0,00125 & 7 & 4 & -3 \\
\hline Switzerland & 0,93 & 0,96 & 0,96 & 0,96 & 0,9525 & 0,947 & $-0,0055$ & 3 & 7 & 4 \\
\hline Japan & 0,95 & 0,94 & 0,947 & 0,94 & 0,94425 & 0,943 & $-0,00125$ & 9 & 11 & 2 \\
\hline Netherlands & 0,89 & 0,95 & 0,959 & 0,99 & 0,94725 & 0,943 & $-0,00425$ & 8 & 11 & 3 \\
\hline France & 0,91 & 0,94 & 0,903 & 0,97 & 0,93075 & 0,938 & 0,00725 & 18 & 16 & -2 \\
\hline Finland & 0,89 & 0,94 & 0,909 & 0,99 & 0,93225 & 0,941 & 0,00875 & 17 & 13 & -4 \\
\hline United States & 0,87 & 0,99 & 0,94 & 0,97 & 0,9425 & 0,944 & 0,0015 & 11 & 10 & -1 \\
\hline Spain & 0,91 & 0,9 & 0,887 & 0,97 & 0,91675 & 0,928 & 0,01125 & 22 & 21 & -1 \\
\hline Denmark & 0,87 & 0,96 & 0,944 & 0,99 & 0,941 & 0,941 & 0 & 13 & 13 & 0 \\
\hline Austria & 0,90 & 0,95 & 0,943 & 0,96 & 0,93825 & 0,936 & $-0,00225$ & 14 & 17 & 3 \\
\hline United Kingdom & 0,89 & 0,94 & 0,95 & 0,99 & 0,9425 & 0,939 & $-0,0035$ & 11 & 15 & 4 \\
\hline Belgium & 0,90 & 0,94 & 0,921 & 0,99 & 0,93775 & 0,945 & 0,00725 & 15 & 9 & -6 \\
\hline Luxembourg & 0,89 & 1 & 0,962 & 0,95 & 0,9505 & 0,949 & $-0,0015$ & 4 & 4 & 0 \\
\hline New Zealand & 0,90 & 0,9 & 0,954 & 0,99 & 0,936 & 0,933 & $-0,003$ & 16 & 19 & 3 \\
\hline Italy & 0,92 & 0,94 & 0,912 & 0,95 & 0,9305 & 0,934 & 0,0035 & 19 & 18 & -1 \\
\hline Germany & 0,90 & 0,94 & 0,909 & 0,96 & 0,92725 & 0,93 & 0,00275 & 20 & 20 & 0 \\
\hline Greece & 0,89 & 0,88 & 0,905 & 0,97 & 0,91125 & 0,912 & 0,00075 & 26 & 24 & -2 \\
\hline Republic of Korea & 0,85 & 0,87 & 0,966 & 0,97 & 0,914 & 0,901 & $-0,013$ & 24 & 27 & 3 \\
\hline Portugal & 0,87 & 0,87 & 0,937 & 0,97 & 0,91175 & 0,904 & $-0,00775$ & 21 & 26 & 5 \\
\hline
\end{tabular}


Employment-adjusted Human Development Index

\begin{tabular}{|c|c|c|c|c|c|c|c|c|c|c|}
\hline & LEI & GDPI & EMPI & EI & E-HDI & HDI & HDI-E-HDI & R(E-HDI) & $\mathbf{R}(\mathrm{HDI})$ & R(HDI-E-HDI) \\
\hline Czech Republic & 0,84 & 0,85 & 0,922 & 0,93 & 0,8855 & 0,874 & $-0,0115$ & 29 & 29 & 0 \\
\hline Hungary & 0,80 & 0,83 & 0,941 & 0,96 & 0,88275 & 0,862 & $-0,02075$ & 30 & 32 & 2 \\
\hline Poland & 0,82 & 0,79 & 0,804 & 0,96 & 0,8435 & 0,858 & 0,0145 & 44 & 33 & -11 \\
\hline Slovakia & 0,82 & 0,82 & 0,825 & 0,91 & 0,84375 & 0,849 & 0,00525 & 43 & 37 & -6 \\
\hline Mexico & 0,83 & 0,75 & 0,975 & 0,85 & 0,85125 & 0,814 & $-0,03725$ & 39 & 45 & 6 \\
\hline Turkey & 0,73 & 0,7 & 0,897 & 0,82 & 0,78675 & 0,75 & $-0,03675$ & 63 & 64 & 1 \\
\hline Estonia & 0,77 & 0,82 & 0,9 & 0,97 & 0,865 & 0,853 & $-0,012$ & 34 & 35 & 1 \\
\hline Georgia & 0,76 & 0,54 & 0,885 & 0,9 & 0,77125 & 0,732 & $-0,03925$ & 65 & 66 & 1 \\
\hline Hong Kong & 0,94 & 0,94 & 0,921 & 0,87 & 0,91775 & 0,916 & $-0,00175$ & 21 & 22 & 1 \\
\hline Israel & 0,91 & 0,88 & 0,893 & 0,95 & 0,90825 & 0,915 & 0,00675 & 27 & 23 & -4 \\
\hline Latvia & 0,78 & 0,77 & 0,894 & 0,96 & 0,851 & 0,836 & $-0,015$ & 40 & 43 & 3 \\
\hline Lithuania & 0,79 & 0,79 & 0,876 & 0,97 & 0,8565 & 0,852 & $-0,0045$ & 36 & 36 & 0 \\
\hline Malaysia & 0,8 & 0,76 & 0,964 & 0,83 & 0,8385 & 0,796 & $-0,0425$ & 45 & 50 & 5 \\
\hline Argentina & 0,82 & 0,8 & 0,846 & 0,96 & 0,8565 & 0,863 & 0,0065 & 36 & 31 & -5 \\
\hline Bulgaria & 0,79 & 0,73 & 0,863 & 0,91 & 0,82325 & 0,808 & $-0,01525$ & 51 & 46 & -5 \\
\hline Chile & 0,88 & 0,77 & 0,926 & 0,91 & 0,8715 & 0,854 & $-0,0175$ & 33 & 34 & 1 \\
\hline Croatia & 0,83 & 0,79 & 0,857 & 0,9 & 0,84425 & 0,841 & $-0,00325$ & 41 & 41 & 0 \\
\hline China & 0,78 & 0,65 & 0,957 & 0,84 & 0,80675 & 0,755 & $-0,05175$ & 54 & 62 & 8 \\
\hline Russia & 0,67 & 0,76 & 0,92 & 0,96 & 0,8275 & 0,795 & $-0,0325$ & 48 & 51 & 3 \\
\hline Belarus & 0,72 & 0,68 & 0,969 & 0,95 & 0,82975 & 0,786 & $-0,04375$ & 47 & 54 & 7 \\
\hline Cuba & 0,87 & 0,67 & 0,977 & 0,91 & 0,85675 & 0,817 & $-0,03975$ & 35 & 44 & 9 \\
\hline Ecuador & 0,82 & 0,6 & 0,885 & 0,86 & 0,79125 & 0,759 & $-0,03225$ & 62 & 60 & -2 \\
\hline Panama & 0,83 & 0,71 & 0,864 & 0,88 & 0,821 & 0,804 & $-0,017$ & 52 & 47 & -5 \\
\hline Romania & 0,77 & 0,72 & 0,93 & 0,89 & 0,8275 & 0,792 & $-0,0355$ & 48 & 52 & 4 \\
\hline Trinidad & 0,75 & 0,78 & 0,895 & 0,88 & 0,82625 & 0,801 & $-0,02525$ & 50 & 48 & -2 \\
\hline Ukraine & 0,69 & 0,67 & 0,909 & 0,95 & 0,80475 & 0,766 & $-0,03875$ & 55 & 57 & 2 \\
\hline Slovakia & 0,82 & 0,82 & 0,826 & 0,91 & 0,844 & 0,849 & 0,005 & 42 & 37 & -5 \\
\hline Paraguay & 0,77 & 0,64 & 0,919 & 0,86 & 0,79725 & 0,755 & $-0,04225$ & 60 & 62 & 2 \\
\hline Uruguay & 0,84 & 0,74 & 0,831 & 0,94 & 0,83775 & 0,84 & 0,00225 & 46 & 42 & -4 \\
\hline South Africa & 0,39 & 0,77 & 0,72 & 0,81 & 0,6725 & 0,658 & $-0,0145$ & 76 & 76 & 0 \\
\hline Malta & 0,89 & 0,86 & 0,924 & 0,85 & 0,881 & 0,867 & $-0,014$ & 32 & 30 & -2 \\
\hline Moldova & 0,71 & 0,45 & 0,919 & 0,85 & 0,73225 & 0,671 & $-0,06125$ & 72 & 73 & 1 \\
\hline Kyrgyzstan & 0,7 & 0,48 & 0,901 & 0,93 & 0,75275 & 0,702 & $-0,05075$ & 68 & 68 & 0 \\
\hline Cyprus & 0,89 & 0,87 & 0,959 & 0,91 & 0,90725 & 0,891 & $-0,01625$ & 28 & 28 & 0 \\
\hline Albania & 0,81 & 0,64 & 0,85 & 0,89 & 0,7975 & 0,78 & $-0,0175$ & 59 & 56 & -3 \\
\hline Bolivia & 0,65 & 0,54 & $\mathrm{n} / \mathrm{a}$ & 0,87 & $\mathrm{n} / \mathrm{a}$ & 0,687 & $\mathrm{n} / \mathrm{a}$ & $\mathrm{n} / \mathrm{a}$ & 72 & $\mathrm{n} / \mathrm{a}$ \\
\hline Kuwait & 0,87 & 0,87 & 0,987 & 0,8 & 0,88175 & 0,844 & $-0,03775$ & 31 & 40 & 9 \\
\hline Nicaragua & 0,75 & 0,58 & 0,923 & 0,74 & 0,74825 & 0,69 & $-0,05825$ & 70 & 71 & 1 \\
\hline Macedonia & 0,81 & 0,7 & 0,633 & 0,87 & 0,75325 & 0,797 & 0,04375 & 67 & 49 & -18 \\
\hline Singapore & 0,89 & 0,92 & 0,941 & 0,91 & 0,91525 & 0,907 & $-0,00825$ & 23 & 25 & 2 \\
\hline Tajikistan & 0,64 & 0,4 & 0,919 & 0,91 & 0,71725 & 0,652 & $-0,06525$ & 75 & 77 & 2 \\
\hline Uzbekistan & 0,69 & 0,48 & 0,831 & 0,91 & 0,72775 & 0,694 & $-0,03375$ & 73 & 70 & -3 \\
\hline Peru & 0,75 & 0,66 & 0,928 & 0,88 & 0,8045 & 0,762 & $-0,0425$ & 56 & 58 & 2 \\
\hline Colombia & 0,79 & 0,7 & 0,856 & 0,86 & 0,8015 & 0,785 & $-0,0165$ & 57 & 55 & -2 \\
\hline Philippines & 0,76 & 0,63 & 0,886 & 0,89 & 0,7915 & 0,758 & $-0,0335$ & 61 & 61 & 0 \\
\hline Indonesia & 0,7 & 0,59 & 0,903 & 0,81 & \begin{tabular}{|l|}
0,75075 \\
\end{tabular} & 0,697 & $-0,05375$ & 69 & 69 & 0 \\
\hline Egypt & 0,75 & 0,61 & 0,89 & 0,62 & 0,7175 & 0,659 & $-0,0585$ & 74 & 75 & 1 \\
\hline El Salvador & 0,76 & 0,65 & 0,931 & 0,76 & 0,77525 & 0,722 & $-0,05325$ & 64 & 67 & 3 \\
\hline
\end{tabular}




\begin{tabular}{|c|c|c|c|c|c|c|c|c|c|c|}
\hline & LEI & GDPI & EMPI & EI & E-HDI & HDI & HDI-E-HDI & R(E-HDI) & R(HDI) & R(HDI-E-HDI) \\
\hline Honduras & 0,71 & 0,55 & 0,949 & 0,74 & 0,73725 & 0,667 & $-0,07025$ & 71 & 74 & 3 \\
\hline Dominican Republic & 0,7 & 0,7 & 0,833 & 0,84 & 0,76825 & 0,749 & $-0,01925$ & 66 & 65 & -1 \\
\hline Bahrain & 0,82 & 0,86 & 0,883 & 0,86 & 0,85575 & 0,846 & $-0,00975$ & 38 & 39 & 1 \\
\hline Kazakhstan & 0,64 & 0,7 & 0,912 & 0,94 & 0,798 & 0,761 & $-0,037$ & 58 & 59 & 1 \\
\hline Brazil & 0,76 & 0,73 & 0,903 & 0,89 & 0,82075 & 0,792 & $-0,02875$ & 53 & 52 & -1 \\
\hline
\end{tabular}

EMPI, E-HDI, R(E-HDI): Authors' own calculations

Source: UNDP (2005); ILO

Table 6: Employment-adjusted Human Development Index (E-HDI): 2003

\begin{tabular}{|c|c|c|c|c|c|c|c|c|c|c|}
\hline & LEI & GDPI & EMPI & EI & E-HDI & HDI & HDI-E-HDI & $R(\mathrm{HDI})$ & R(E-HDI) & R(HDI-E-HDI) \\
\hline Iceland & 0,93 & 0,98 & 0,975 & 0,97 & 0,96375 & 0,96 & $-0,00375$ & 2 & 1 & 1 \\
\hline Norway & 0,91 & 0,99 & 0,954 & 0,99 & 0,961 & 0,965 & 0,004 & 1 & 2 & -1 \\
\hline Australia & 0,92 & 0,95 & 0,949 & 0,99 & 0,95225 & 0,957 & 0,00475 & 3 & 4 & -1 \\
\hline Canada & 0,92 & 0,96 & 0,932 & 0,97 & 0,9455 & 0,95 & 0,0045 & 6 & 10 & -4 \\
\hline Ireland & 0,88 & 1 & 0,957 & 0,99 & 0,95675 & 0,956 & $-0,00075$ & 4 & 3 & 1 \\
\hline Sweden & 0,92 & 0,95 & 0,944 & 0,98 & 0,9485 & 0,951 & 0,0025 & 5 & 8 & -3 \\
\hline Switzerland & 0,93 & 0,97 & 0,959 & 0,95 & 0,95225 & 0,947 & $-0,00525$ & 9 & 4 & 5 \\
\hline Japan & 0,95 & 0,95 & 0,956 & 0,94 & 0,949 & 0,949 & 0 & 7 & 7 & 0 \\
\hline Netherlands & 0,89 & 0,96 & 0,938 & 0,99 & 0,9445 & 0,947 & 0,0025 & 9 & 11 & -2 \\
\hline France & 0,91 & 0,95 & 0,9 & 0,97 & 0,9325 & 0,942 & 0,0095 & 16 & 19 & -3 \\
\hline Finland & 0,89 & 0,95 & 0,914 & 0,99 & 0,936 & 0,947 & 0,011 & 9 & 17 & -8 \\
\hline United States & 0,88 & 1 & 0,949 & 0,97 & 0,94975 & 0,948 & $-0,00175$ & 8 & 6 & 2 \\
\hline Spain & 0,91 & 0,92 & 0,909 & 0,98 & 0,92975 & 0,938 & 0,00825 & 19 & 21 & -2 \\
\hline Denmark & 0,87 & 0,96 & 0,951 & 0,99 & 0,94275 & 0,943 & 0,00025 & 15 & 13 & 2 \\
\hline Austria & 0,90 & 0,96 & 0,942 & 0,96 & 0,9405 & 0,944 & 0,0035 & 14 & 15 & -1 \\
\hline United Kingdom & 0,89 & 0,96 & 0,952 & 0,97 & 0,943 & 0,94 & $-0,003$ & 18 & 12 & 6 \\
\hline Belgium & 0,90 & 0,96 & 0,916 & 0,98 & 0,939 & 0,945 & 0,006 & 12 & 16 & -4 \\
\hline Luxembourg & 0,89 & 1 & 0,954 & 0,94 & 0,946 & 0,945 & $-0,001$ & 12 & 9 & 3 \\
\hline New Zealand & 0,90 & 0,91 & 0,964 & 0,99 & 0,941 & 0,936 & $-0,005$ & 20 & 14 & 6 \\
\hline Italy & 0,92 & 0,94 & 0,923 & 0,96 & 0,93575 & 0,94 & 0,00425 & 18 & 18 & 0 \\
\hline Germany & 0,90 & 0,9 & 0,907 & 0,96 & 0,91675 & 0,932 & 0,01525 & 21 & 26 & -5 \\
\hline Greece & 0,89 & 0,9 & 0,894 & 0,97 & 0,9135 & 0,921 & 0,0075 & 24 & 27 & -3 \\
\hline Republic of Korea & 0,87 & 0,89 & 0,962 & 0,98 & 0,9255 & 0,912 & $-0,0135$ & 26 & 22 & 4 \\
\hline Portugal & 0,87 & 0,88 & 0,925 & 0,96 & 0,90875 & 0,904 & $-0,00475$ & 27 & 28 & -1 \\
\hline Czech Republic & 0,85 & 0,88 & 0,92 & 0,93 & 0,895 & 0,885 & $-0,01$ & 29 & 30 & -1 \\
\hline Hungary & 0,80 & 0,86 & 0,929 & 0,95 & 0,88475 & 0,869 & $-0,01575$ & 32 & 32 & 0 \\
\hline Poland & 0,83 & 0,81 & 0,822 & 0,95 & 0,853 & 0,862 & 0,009 & 34 & 42 & -8 \\
\hline Slovakia & 0,82 & 0,83 & 0,836 & 0,92 & 0,8515 & 0,856 & 0,0045 & 39 & 43 & -4 \\
\hline Mexico & 0,84 & 0,77 & 0,964 & 0,86 & 0,8585 & 0,821 & $-0,0375$ & 45 & 39 & 6 \\
\hline Turkey & 0,73 & 0,73 & 0,9 & 0,81 & 0,7925 & 0,757 & $-0,0355$ & 63 & 63 & 0 \\
\hline Estonia & 0,78 & 0,83 & 0,903 & 0,97 & 0,87075 & 0,858 & $-0,01275$ & 37 & 35 & 2 \\
\hline Georgia & 0,76 & 0,56 & 0,874 & 0,91 & 0,776 & 0,743 & $-0,033$ & 66 & 65 & 1 \\
\hline Hong Kong & 0,95 & 0,96 & 0,932 & 0,88 & 0,9305 & 0,927 & $-0,0035$ & 22 & 20 & 2 \\
\hline Israel & 0,92 & 0,92 & 0,896 & 0,95 & 0,9215 & 0,927 & 0,0055 & 22 & 24 & -2 \\
\hline Latvia & 0,78 & 0,79 & 0,896 & 0,96 & 0,8565 & 0,845 & $-0,0115$ & 43 & 40 & 3 \\
\hline Lithuania & 0,79 & 0,81 & 0,886 & 0,97 & 0,864 & 0,857 & $-0,007$ & 38 & 38 & 0 \\
\hline Malaysia & 0,81 & 0,77 & 0,965 & 0,84 & 0,84625 & 0,805 & $-0,04125$ & 49 & 46 & 3 \\
\hline Argentina & 0,83 & 0,82 & 0,874 & 0,95 & 0,8685 & 0,863 & $-0,0055$ & 33 & 36 & -3 \\
\hline
\end{tabular}




\begin{tabular}{|c|c|c|c|c|c|c|c|c|c|c|}
\hline & LEI & GDPI & EMPI & EI & E-HDI & HDI & HDI-E-HDI & $\mathbf{R}(\mathrm{HDI})$ & R(E-HDI) & R(HDI-E-HDI \\
\hline Bulgaria & 0,79 & 0,73 & 0,88 & 0,92 & 0,83 & 0,816 & $-0,014$ & 46 & 50 & -4 \\
\hline Chile & 0,89 & 0,78 & 0,922 & 0,91 & 0,8755 & 0,859 & $-0,0165$ & 35 & 34 & 1 \\
\hline Croatia & 0,84 & 0,8 & 0,862 & 0,9 & 0,8505 & 0,846 & $-0,0045$ & 42 & 44 & -2 \\
\hline China & 0,78 & 0,68 & 0,958 & 0,84 & 0,8145 & 0,768 & $-0,0465$ & 59 & 54 & 5 \\
\hline Russia & 0,67 & 0,77 & 0,922 & 0,95 & 0,828 & 0,797 & $-0,031$ & 51 & 51 & 0 \\
\hline Belarus & 0,72 & 0,71 & 0,981 & 0,95 & 0,84025 & 0,794 & $-0,04625$ & 53 & 47 & 6 \\
\hline Cuba & 0,88 & 0,67 & 0,981 & 0,93 & 0,86525 & 0,826 & $-0,03925$ & 44 & 37 & 7 \\
\hline Ecuador & 0,82 & 0,61 & 0,914 & 0,86 & 0,801 & 0,765 & $-0,036$ & 61 & 61 & 0 \\
\hline Panama & 0,83 & 0,72 & 0,876 & 0,88 & 0,8265 & 0,809 & $-0,0175$ & 47 & 56 & -9 \\
\hline Romania & 0,78 & 0,74 & 0,92 & 0,9 & 0,835 & 0,805 & $-0,03$ & 49 & 49 & 0 \\
\hline Trinidad & 0,75 & 0,8 & 0,917 & 0,88 & 0,83675 & 0,809 & $-0,02775$ & 47 & 48 & -1 \\
\hline Ukraine & 0,69 & 0,69 & 0,914 & 0,94 & 0,8085 & 0,774 & $-0,0345$ & 58 & 57 & 1 \\
\hline Slovakia & 0,82 & 0,83 & 0,819 & 0,92 & 0,84725 & 0,856 & 0,00875 & 39 & 45 & -6 \\
\hline Paraguay & 0,77 & 0,65 & 0,926 & 0,86 & 0,8015 & 0,757 & $-0,0445$ & 63 & 59 & 4 \\
\hline Uruguay & 0,84 & 0,76 & 0,869 & 0,95 & 0,85475 & 0,851 & $-0,00375$ & 41 & 41 & 0 \\
\hline South Africa & 0,37 & 0,79 & 0,738 & 0,8 & 0,6745 & 0,653 & $-0,0215$ & 76 & 76 & 0 \\
\hline Malta & 0,89 & 0,87 & 0,928 & 0,86 & 0,887 & 0,875 & $-0,012$ & 31 & 31 & 0 \\
\hline Moldova & 0,72 & 0,48 & 0,927 & 0,89 & 0,75425 & 0,694 & $-0,06025$ & 73 & 71 & 2 \\
\hline Kyrgyzstan & 0,7 & 0,49 & 0,915 & 0,92 & 0,75625 & 0,705 & $-0,05125$ & 69 & 69 & 0 \\
\hline Cyprus & 0,9 & 0,91 & 0,953 & 0,91 & 0,91825 & 0,903 & $-0,01525$ & 28 & 25 & 3 \\
\hline Albania & 0,82 & 0,65 & 0,856 & 0,88 & 0,8015 & 0,784 & $-0,0175$ & 56 & 59 & -3 \\
\hline Bolivia & 0,65 & 0,55 & 0,938 & 0,87 & 0,752 & 0,692 & $-0,06$ & 74 & 72 & 2 \\
\hline Kuwait & 0,87 & 0,88 & 0,986 & 0,87 & 0,9015 & 0,875 & $-0,0265$ & 30 & 29 & 1 \\
\hline Nicaragua & 0,75 & 0,6 & 0,935 & 0,75 & 0,75875 & 0,698 & $-0,06075$ & 71 & 67 & 4 \\
\hline Macedonia & 0,82 & 0,7 & 0,628 & 0,87 & 0,7545 & 0,796 & 0,0415 & 52 & 70 & -18 \\
\hline Singapore & 0,9 & 0,94 & 0,942 & 0,91 & 0,923 & 0,916 & $-0,007$ & 25 & 23 & 2 \\
\hline Tajikistan & 0,65 & 0,41 & 0,84 & 0,9 & 0,7 & 0,652 & $-0,048$ & 77 & 75 & 2 \\
\hline Uzbekistan & 0,69 & 0,49 & $\mathrm{n} / \mathrm{a}$ & 0,91 & $n / a$ & 0,696 & $\mathrm{n} / \mathrm{a}$ & 72 & $\mathrm{n} / \mathrm{a}$ & $\mathrm{n} / \mathrm{a}$ \\
\hline Peru & 0,75 & 0,67 & 0,926 & 0,87 & 0,804 & 0,767 & $-0,037$ & 60 & 58 & 2 \\
\hline Colombia & 0,79 & 0,72 & 0,872 & 0,86 & 0,8105 & 0,79 & $-0,0205$ & 55 & 55 & 0 \\
\hline Philippines & 0,76 & 0,64 & 0,882 & 0,89 & 0,793 & 0,763 & $-0,03$ & 62 & 62 & 0 \\
\hline Indonesia & 0,7 & 0,6 & 0,901 & 0,83 & 0,75775 & 0,711 & $-0,04675$ & 68 & 68 & 0 \\
\hline Egypt & 0,75 & 0,62 & 0,897 & 0,73 & 0,74925 & 0,702 & $-0,04725$ & 70 & 73 & -3 \\
\hline El Salvador & 0,77 & 0,65 & 0,932 & 0,76 & 0,778 & 0,729 & $-0,049$ & 67 & 64 & 3 \\
\hline Honduras & 0,72 & 0,56 & 0,941 & 0,77 & 0,74775 & 0,683 & $-0,06475$ & 75 & 74 & 1 \\
\hline Dominican Republic & 0,71 & 0,72 & 0,816 & 0,83 & 0,769 & 0,751 & $-0,018$ & 65 & 66 & -1 \\
\hline Bahrain & 0,82 & 0,89 & 0,937 & 0,86 & 0,87675 & 0,859 & $-0,01775$ & 35 & 33 & 2 \\
\hline Kazakhstan & 0,64 & 0,72 & 0,916 & 0,96 & 0,809 & 0,774 & $-0,035$ & 57 & 56 & 1 \\
\hline Brazil & 0,76 & 0,74 & 0,911 & 0,88 & 0,82275 & 0,792 & $-0,03075$ & 54 & 53 & 1 \\
\hline
\end{tabular}

EMPI, E-HDI, R(E-HDI): Authors' own calculations

Source: UNDP (2006); ILO

Table 7: Employment-adjusted Human Development Index (E-HDI): 2004 


\begin{tabular}{|c|c|c|c|c|c|c|c|c|c|c|}
\hline & LEI & GDPI & EMPI & EI & E-HDI & HDI & HDI-E-HDI & $R$ (HDI) & $R$ (E-HDI) & R(HDI-E-HDI) \\
\hline Iceland & 0,94 & 0,985 & 0,97 & 0,978 & 0,9685 & 0,968 & $-0,0005$ & 1 & 1 & 0 \\
\hline Norway & 0,91 & 1 & 0,965 & 0,991 & 0,96725 & 0,968 & 0,00075 & 1 & 2 & -1 \\
\hline Australia & 0,93 & 0,962 & 0,951 & 0,993 & 0,95925 & 0,962 & 0,00275 & 3 & 3 & 0 \\
\hline Canada & 0,92 & 0,97 & 0,937 & 0,991 & 0,95475 & 0,961 & 0,00625 & 4 & 6 & -2 \\
\hline Ireland & 0,89 & 0,994 & 0,956 & 0,993 & 0,95825 & 0,959 & 0,00075 & 5 & 4 & 1 \\
\hline Sweden & 0,92 & 0,965 & 0,93 & 0,978 & 0,94925 & 0,956 & 0,00675 & 6 & 11 & -5 \\
\hline Switzerland & 0,94 & 0,981 & 0,96 & 0,946 & 0,95625 & 0,955 & $-0,00125$ & 7 & 5 & 2 \\
\hline Japan & 0,95 & 0,959 & 0,959 & 0,946 & 0,9545 & 0,953 & $-0,0015$ & 8 & 8 & 0 \\
\hline Netherlands & 0,90 & 0,966 & 0,961 & 0,988 & 0,95475 & 0,953 & $-0,00175$ & 8 & 6 & 2 \\
\hline France & 0,92 & 0,954 & 0,906 & 0,982 & 0,94025 & 0,952 & 0,01175 & 10 & 18 & -8 \\
\hline Finland & 0,90 & 0,964 & 0,923 & 0,993 & 0,9445 & 0,952 & 0,0075 & 10 & 16 & -6 \\
\hline United States & 0,88 & 1 & 0,954 & 0,971 & 0,9515 & 0,951 & $-0,0005$ & 12 & 10 & 2 \\
\hline Spain & 0,93 & 0,935 & 0,915 & 0,987 & 0,9405 & 0,949 & 0,0085 & 13 & 17 & -4 \\
\hline Denmark & 0,88 & 0,973 & 0,961 & 0,993 & 0,952 & 0,949 & $-0,003$ & 13 & 9 & 4 \\
\hline Austria & 0,91 & 0,971 & 0,952 & 0,966 & 0,949 & 0,948 & $-0,001$ & 15 & 12 & 3 \\
\hline United Kingdom & 0,90 & 0,969 & 0,947 & 0,97 & 0,9465 & 0,946 & $-0,0005$ & 16 & 14 & 2 \\
\hline Belgium & 0,90 & 0,963 & 0,918 & 0,977 & 0,93875 & 0,946 & 0,00725 & 16 & 20 & -4 \\
\hline Luxembourg & 0,89 & 1 & 0,952 & 0,942 & 0,94625 & 0,944 & $-0,00225$ & 18 & 15 & 3 \\
\hline New Zealand & 0,91 & 0,922 & 0,962 & 0,993 & 0,9475 & 0,943 & $-0,0045$ & 19 & 13 & 6 \\
\hline Italy & 0,92 & 0,944 & 0,932 & 0,958 & 0,939 & 0,941 & 0,002 & 20 & 19 & 1 \\
\hline Germany & 0,90 & 0,949 & 0,916 & 0,953 & 0,93 & 0,935 & 0,005 & 22 & 23 & -1 \\
\hline Greece & 0,90 & 0,91 & 0,911 & 0,97 & 0,92225 & 0,926 & 0,00375 & 24 & 26 & -2 \\
\hline Republic of Korea & 0,88 & 0,9 & 0,965 & 0,98 & 0,93175 & 0,921 & $-0,01075$ & 26 & 22 & 4 \\
\hline Portugal & 0,88 & 0,888 & 0,923 & 0,925 & 0,90375 & 0,897 & $-0,00675$ & 28 & 29 & -1 \\
\hline Czech Republic & 0,85 & 0,889 & 0,928 & 0,936 & 0,9005 & 0,891 & $-0,0095$ & 29 & 30 & -1 \\
\hline Hungary & 0,80 & 0,866 & 0,925 & 0,958 & 0,887 & 0,874 & $-0,013$ & 32 & 32 & 0 \\
\hline Poland & 0,84 & 0,823 & 0,862 & 0,951 & 0,868 & 0,87 & 0,002 & 33 & 40 & -7 \\
\hline Slovakia & 0,82 & 0,846 & 0,866 & 0,921 & 0,8635 & 0,863 & $-0,0005$ & 37 & 43 & -6 \\
\hline Mexico & 0,84 & 0,781 & 0,968 & 0,863 & 0,86375 & 0,829 & $-0,03475$ & 45 & 41 & 4 \\
\hline Turkey & 0,77 & 0,74 & 0,901 & 0,812 & 0,8065 & 0,775 & $-0,0315$ & 61 & 62 & -1 \\
\hline Estonia & 0,77 & 0,842 & 0,95 & 0,968 & 0,8825 & 0,86 & $-0,0225$ & 40 & 35 & 5 \\
\hline Georgia & 0,761 & 0,587 & 0,862 & 0,914 & 0,781 & 0,754 & $-0,027$ & 66 & 66 & 0 \\
\hline Hong Kong & 0,949 & 0,977 & 0,944 & 0,885 & 0,93875 & 0,937 & $-0,00175$ & 21 & 20 & 1 \\
\hline Israel & 0,921 & 0,927 & 0,91 & 0,946 & 0,926 & 0,932 & 0,006 & 23 & 25 & -2 \\
\hline Latvia & 0,784 & 0,821 & 0,913 & 0,961 & 0,86975 & 0,855 & $-0,01475$ & 41 & 39 & 2 \\
\hline Lithuania & 0,792 & 0,831 & 0,917 & 0,965 & 0,87625 & 0,862 & $-0,01425$ & 39 & 36 & 3 \\
\hline Malaysia & 0,811 & 0,783 & 0,965 & 0,839 & 0,8495 & 0,811 & $-0,0385$ & 50 & 47 & 3 \\
\hline Argentina & 0,836 & 0,815 & 0,905 & 0,946 & 0,8755 & 0,869 & $-0,0065$ & 34 & 37 & -3 \\
\hline Bulgaria & 0,795 & 0,752 & 0,91 & 0,926 & 0,84575 & 0,824 & $-0,02175$ & 46 & 48 & -2 \\
\hline Chile & 0,889 & 0,799 & 0,94 & 0,914 & 0,8855 & 0,867 & $-0,0185$ & 35 & 33 & 2 \\
\hline Croatia & 0,839 & 0,813 & 0,889 & 0,899 & 0,86 & 0,85 & $-0,01$ & 43 & 45 & -2 \\
\hline China & 0,792 & 0,703 & 0,959 & 0,837 & 0,82275 & 0,777 & $-0,04575$ & 60 & 56 & 4 \\
\hline Russia & 0,667 & 0,782 & 0,928 & 0,956 & 0,83325 & 0,802 & $-0,03125$ & 52 & 52 & 0 \\
\hline Belarus & 0,728 & 0,73 & 0,988 & 0,956 & 0,8505 & 0,804 & $-0,0465$ & 51 & 46 & 5 \\
\hline Cuba & 0,879 & 0,683 & 0,981 & 0,952 & 0,87375 & 0,838 & $-0,03575$ & 44 & 38 & 6 \\
\hline Ecuador & 0,828 & 0,629 & 0,922 & 0,858 & 0,80925 & 0,772 & $-0,03725$ & 63 & 60 & 3 \\
\hline Panama & 0,836 & 0,723 & 0,909 & 0,878 & 0,8365 & 0,812 & $-0,0245$ & 49 & 51 & -2 \\
\hline
\end{tabular}




\begin{tabular}{|c|c|c|c|c|c|c|c|c|c|c|}
\hline & LEI & GDPI & EMPI & EI & E-HDI & HDI & HDI-E-HDI & $\mathbf{R}(\mathrm{HDI})$ & R(E-HDI) & R(HDI-E-HDI) \\
\hline Romania & 0,782 & 0,752 & 0,927 & 0,905 & 0,8415 & 0,813 & $-0,0285$ & 48 & 50 & -2 \\
\hline Trinidad & 0,737 & 0,832 & 0,938 & 0,872 & 0,84475 & 0,814 & $-0,03075$ & 47 & 49 & -2 \\
\hline Ukraine & 0,711 & 0,705 & 0,932 & 0,948 & 0,824 & 0,788 & $-0,036$ & 58 & 55 & 3 \\
\hline Slovakia & 0,821 & 0,846 & 0,867 & 0,921 & 0,86375 & 0,863 & $-0,00075$ & 37 & 41 & -4 \\
\hline Paraguay & 0,771 & 0,641 & 0,933 & 0,853 & 0,7995 & 0,755 & $-0,0445$ & 65 & 63 & 2 \\
\hline Uruguay & 0,848 & 0,768 & 0,894 & 0,942 & 0,863 & 0,852 & $-0,011$ & 42 & 44 & -2 \\
\hline South Africa & 0,43 & 0,786 & 0,745 & 0,806 & 0,69175 & 0,674 & $-0,01775$ & 76 & 77 & -1 \\
\hline Malta & 0,901 & 0,877 & 0,927 & 0,856 & 0,89025 & 0,878 & $-0,01225$ & 31 & 31 & 0 \\
\hline Moldova & 0,724 & 0,508 & 0,926 & 0,892 & 0,7625 & 0,708 & $-0,0545$ & 70 & 70 & 0 \\
\hline Kyrgyzstan & 0,676 & 0,494 & 0,919 & 0,917 & 0,7515 & 0,696 & $-0,0555$ & 74 & 73 & 1 \\
\hline Cyprus & 0,9 & 0,905 & 0,955 & 0,904 & 0,916 & 0,903 & $-0,013$ & 27 & 27 & 0 \\
\hline Albania & 0,853 & 0,663 & 0,862 & 0,887 & 0,81625 & 0,801 & $-0,01525$ & 53 & 57 & -4 \\
\hline Bolivia & 0,662 & 0,557 & 0,92 & 0,865 & 0,751 & 0,695 & $-0,056$ & 75 & 74 & 1 \\
\hline Kuwait & 0,871 & 0,93 & 0,985 & 0,871 & 0,91425 & 0,891 & $-0,02325$ & 29 & 28 & 1 \\
\hline Nicaragua & 0,782 & 0,601 & 0,944 & 0,747 & 0,7685 & 0,71 & $-0,0585$ & 69 & 68 & 1 \\
\hline Macedonia & 0,814 & 0,714 & 0,627 & 0,875 & 0,7575 & 0,801 & 0,0435 & 53 & 71 & -18 \\
\hline Singapore & 0,907 & 0,95 & 0,955 & 0,908 & 0,93 & 0,922 & $-0,008$ & 25 & 23 & 2 \\
\hline Tajikistan & 0,689 & 0,435 & 0,81 & 0,896 & 0,7075 & 0,673 & $-0,0345$ & 77 & 76 & 1 \\
\hline Uzbekistan & 0,696 & 0,505 & 0,894 & 0,906 & 0,75025 & 0,702 & $-0,04825$ & 72 & 75 & -3 \\
\hline Peru & 0,761 & 0,684 & 0,928 & 0,872 & 0,81125 & 0,773 & $-0,03825$ & 62 & 59 & 3 \\
\hline Colombia & 0,788 & 0,716 & 0,873 & 0,869 & 0,8115 & 0,791 & $-0,0205$ & 57 & 58 & -1 \\
\hline Philippines & 0,767 & 0,657 & 0,92 & 0,888 & 0,808 & 0,771 & $-0,037$ & 64 & 61 & 3 \\
\hline Indonesia & 0,745 & 0,609 & 0,897 & 0,83 & 0,77025 & 0,728 & $-0,04225$ & 68 & 67 & 1 \\
\hline Egypt & 0,761 & 0,629 & 0,894 & 0,732 & 0,754 & 0,708 & $-0,046$ & 70 & 72 & -2 \\
\hline El Salvador & 0,772 & 0,661 & 0,934 & 0,772 & 0,78475 & 0,735 & $-0,04975$ & 67 & 65 & 2 \\
\hline Honduras & 0,739 & 0,59 & 0,969 & 0,771 & 0,76725 & 0,7 & $-0,06725$ & 73 & 69 & 4 \\
\hline Dominican Republic & 0,776 & 0,736 & 0,84 & 0,827 & 0,79475 & 0,779 & $-0,01575$ & 59 & 64 & -5 \\
\hline Bahrain & 0,837 & 0,896 & 0,936 & 0,864 & 0,88325 & 0,866 & $-0,01725$ & 36 & 34 & 2 \\
\hline Kazakhstan & 0,682 & 0,728 & 0,919 & 0,973 & 0,8255 & 0,794 & $-0,0315$ & 56 & 54 & 2 \\
\hline Brazil & 0,779 & 0,74 & 0,916 & 0,883 & 0,8295 & 0,8 & $-0,0295$ & 55 & 53 & 2 \\
\hline
\end{tabular}

EMPI, E-HDI, R(E-HDI): Authors' own calculations

Source: UNDP (2007-8); ILO

Table 8: Employment-adjusted Human Development Index (E-HDI): 2005-6 